\title{
Interaction between transcription factors PAX6/PAX6-5a and specific members of miR-183-96-182 cluster, may contribute to glioma progression in glioblastoma cell lines
}

\author{
EVANGELOS PAVLAKIS ${ }^{1,5,6}$, ANTON B. TONCHEV ${ }^{1,2}$, ARA KAPRELYAN $^{3}$, \\ YAVOR ENCHEV $^{4}$ and ANASTASSIA STOYKOVA ${ }^{1,5}$
}

\begin{abstract}
${ }^{1}$ Research Group Molecular Developmental Neurobiology, Max-Planck Institute for Biophysical Chemistry, 37077 Göttingen, Germany; Departments of ${ }^{2}$ Anatomy, Histology and Embryology, ${ }^{3}$ Neurology, Medical University of Varna; ${ }^{4}$ Neurosurgery Clinic, University Hospital of the Medical University of Varna, 9002 Varna, Bulgaria; ${ }^{5}$ Cluster of Excellence 'Nanoscale Microscopy and Molecular Physiology of the Brain (CNMPB)', University of Göttingen, 37073 Göttingen, Germany
\end{abstract}

Received August 16, 2016; Accepted January 2, 2017

DOI: 10.3892/or.2017.5411

\begin{abstract}
A misbalance between proliferation and differentiation of neural stem cells in niches for adult brain neurogenesis is a key mechanism in glioma pathogenesis. In the adult brain, the expression of Pax6 marks stem cells in the forebrain neurogenic niche. We analyzed the expression profile of the two active in vertebrates Pax6 isoforms, Pax6 and Pax6-5a, along with the expression of microRNA cluster miR-183-96182 in a large set of glioma patient specimens and glioma cell lines which showed opposite expression level, low and high, respectively, with the progression of tumor malignancy. Our results from biochemical and in vitro studies in glioma cell lines disclosed a specific regulation of the PAX6-5a isoform by miR-183. Mechanistically, we show that the downregulation of the lipid kinase SPHK1 by both PAX6 isoforms and the simultaneous induction of $C T N D D 2$ expression, specifically by PAX6-5a, results in reduced glioma cell survival, decreased migration and invasion and increased cell death, in glioma cell lines. Taken together, our findings point towards the important role of PAX6 and define PAX6-5a as a new essential player in glioma development. Finally, we propose that the expression level of TFs PAX6/PAX6-5a and miR-183-96-182 may potentially serve as prognostic markers for the progression of glioma tumors from low- to high-grade with a potential to identify new therapeutic approaches.
\end{abstract}

Correspondence to: Dr Evangelos Pavlakis, ${ }^{6}$ Present address: Philipps-Universität Marburg, Institut für Molekulare Onkologie, und Genomics Core Facility, Zentrum für Tumor- und Immunbiologie, Hans-Meerwein-Str. 3, D-35043 Marburg, Germany

E-mail: pavlakisvaggelis@gmail.com or evangelos.pavlakis@imt.uni-marburg.de

Key words: miR-183-96-182, PAX6, PAX6-5a, glioma, glioblastoma multiform

\section{Introduction}

Among the four main histopathological types of gliomas, glioblastoma multiforme (GBM, grade IV astrocytoma) is the most aggressive subtype, leading to death within 12-15 months $(1,2)$. The most common oncogenic events encountered in GBM include, amplification of the epidermal growth factor receptor $(E G F R)(3,4)$; overexpression (OE) of components of the platelet derived growth factor (PDGF) pathway (PDGF-A, PDGF-B, PDGFR- $\alpha$ and PDGFR- $\beta$ ) $(4,5)$; mutations of genes residing in chromosome 10, including the tumor suppressor phosphatase and tensin homolog (PTEN) $(4,6,7)$. Recent evidence suggests however that stem and/or progenitors cells in the adult brain may give rise to 'glioma stem cells' (GSCs) representing transformed normal neural stem cells (NSCs) (8-10). These cells are descendents of the bipotent radial glial cells (RGCs), acting as the main progenitor cell type in the developing cortex that are specified and controlled by the expression of the transcription factor (TF) Pax6 (11-16,29). In vertebrate CNS, Pax6 exists in three isoforms; the canonical Pax6, a longer Pax6-5a isoform and the paired-less Pax6 form $(\Delta \mathrm{PD})$. Previous functional in vivo analyses suggested that, while canonical Pax6 regulates neurogenesis, cell proliferation, and patterning in developing telencephalon, the spliced Pax6-5a isoform affects specifically cell proliferation (17). However, in murine embryonic stem cells in vitro, Pax6-5a strongly stimulates neuronal differentiation, while Pax6 failed to show such a robust influence (18).

In humans with GBM, low PAX6 level is considered a negative prognostic marker for the patient $(19,20)$. In highly malignant tumors, the low level of PAX6 correlates with the elevated expression of vascular endothelial growth factor (VEGF), while $P A X 6$ overexpression (OE) in subcutaneous xenographs suppresses the formation of new blood vessels (19). Along the same track, in glioma cell lines, a stable OE of PAX6 suppresses the tumorigenic potential and improves significantly the survival of nude mice, intra-cranially implanted with these cells (20). Together with the reported strong dose-dependent effect for controlling the balance 
between NSC self-renewal and neurogenesis (21), TF Pax6 is a good candidate that may also act as a mediator of neurogenic versus certain aspects of tumorigenic capacity of NSC in the adult brain.

MicroRNAs (miRNAs) have been identified as 'tools' for the in vivo regulation of the endogenous expression level of molecular determinants that are acting as tumor suppressor genes or oncogenes $(22,23)$. Hierarchical clustering analysis based on the expression profiles of 71 miRNAs in human gliomas defined similar signatures in brain tumors and normal neural stem cells (NSCs), suggesting that specific miRNAs may enforce tumorigenesis at the expense of normal brain neurogenesis (24). The clusters/families that displayed NSC-like miRNA signatures include miR-17, miR183-96-182, miR367-302, miR371-373 (24). miR-183-96-182 is a polycistronic, paralogous cluster of miRNAs, which have initially been identified as sensory organ specific $(25,26)$, but also shown to be upregulated in brain cancer (27-32). In glioma tumor biopsies, miR-182 showed specifically elevated expression (33) with the highest level attained in GBM (grade IV) (33). miR-183 has been associated with the targeting of isocitrate dehydrogenase 2 (IDH2) in glioma (34), while knockdown (KD) of the whole miRNA cluster induced oxidative apoptosis thus inhibiting the survival of glioma cells in vitro (35).

Herein, we show that all members of the miR-183-96-182 cluster are highly upregulated in 29 human glioma tumor biopsies and 3 glioma cell lines, which correlates with a simultaneous dramatic reduction of both PAX6 and PAX6-5a isoforms, implying that they could be used as novel diagnostic tools for high-grade glioma. We disclose the preferential regulation of PAX6-5a expression by miR-183, while the canonical PAX6 expression is more strongly affected by both miR-182 and miR-96. Mechanistically, we demonstrate that the tumor suppressive potential of the increased PAX6/PAX6-5a 'dose', could be a result of down regulation of SPHK1 and CTNND2, two key factors for glioma progression, leading to decreased proliferation and increase death of GBM cells, in vitro.

\section{Materials and methods}

$R N A$ extraction and $q R T-P C R$. Total RNA was purified from cell cultures using TRIzol reagent according to manufacturer's instructions (Invitrogen). For mature miRNA expression analysis, cDNA was synthesized using the TaqMan MicroRNA Reverse Transcription kit (Applied Biosystems) and 50-100 ng of total RNA along with miRNA specific stem loop primer, supplied with the TaqMan microRNA Assays kit (Applied Biosystems). The assays used were: hsa-miR-183 (002269; Applied Biosystems),hsa-miR-96(000186; Applied Biosystems) and hsa-miR-182 (002334; Applied Biosystems). qPCR was performed with TaqMan PCR Master Mix according to the manufacturer's instructions and U6 snRNA (001973; Applied Biosystems) or snoRNA202 TaqMan (001232; Applied Biosystems) probes were used as endogenous controls. For gene expression analysis, cDNA was synthesized using the QuantiTect Reverse Transcription kit (Qiagen) according to manufacturer's instructions and 100-150 ng of total RNA. qPCR was performed with GoTaq ${ }^{\circledR}$ qPCR Master Mix (Promega). Primer sequences of target genes were as follows; Pax6-forward: 5'-gaatcagagaagacaggcca-3'; Pax6-reverse: 5'-gtgtaggtatcataactccg-3'; Pax6(5a)-forward: 5'-gagtgaatcag ctcggtgg-3'; Pax6(5a)-reverse 1: 5'-ggacttttgcatctgcatgg-3'; Pax6(5a)-Reverse 2: 5'-cgttttgattgtccagcac-3'; $\beta$-actin-Forward: 5'-tccttcctgggcatggagt-3'; $\beta$-actin-Reverse: 5'-aaagccatgc caatctcatc-3'. Also the following Quantitect primer assays were purchased from Qiagen and used according to manufacturer's advice:Hs_PDGFRA_1_SG (QT00012719),Hs_SPHK1_1_SG (QT01011927), Hs_PDGFRB_1_SG (QT00082327), Hs CTNND1_1_SG (QT00033831), Hs_PAX6_1_SG (QT00071169), Hs_PDGFA_1_SG (QT01664488), Hs_ TAM1_1_SG (QT01009568), Hs_GADPH_1_SG (QT00079247), Hs_PDFGB_1_SG (QT00001260), Hs_ ACTB_1_SG (QT00095431), Hs_NF1_1_SG (QT00065016). In all cases, qPCR was performed on a Realplex2 Mastercycler (Eppendorf).

miRNA expression vectors, mimics and inhibitors. The pre-miR-182, pre-miR-183 and pre-miR-96 sequences were amplified by PCR from human genomic DNA, using the following oligos; miR-182-For: 5'-ctgtgggaagagcgecetc-3'; miR-182-Rev: 5'-caccgagaagaggtcgacttc-3'; miR-183-For: 5'-gagctggtgaggagggttgc-3'; miR-183-Rev: 5'-ccaagcagatg gtactggaac-3'; miR-96-For: 5'-gcaggctggagagtgtgactc-3'; miR96-Rev: 5'-caggcagtgaaaggtgatctg-3'. The products were purified and cloned into the pCIG2 expression vector (CMV-EGFP) upstream of the EGFP sequence. The expression of mature miRNA was verified by qRT-PCR, with TaqMan MicroRNA assays. The following commercial miRNA mimics and inhibitors were purchased from Qiagen and were used according to the manufacturer's advice; miScript miRNA mimics: Syn-hsa-miR-183 (MSY0000261), Syn-hsa-miR-182 (MSY0000259), Syn-hsa-miR-96-5p (MSY0000095). miScript miRNA inhibitors: anti-hsa-96-5p (MIN0000095), anti-hsamiR-183-5p (MIN0000261) and anti-hsa-miR-182-5p (MIN0000259).

Immunocytofluorescence (ICF). For ICF, the cells were seeded on Lab-Tek ${ }^{\circledR}$ II Chamber Slide ${ }^{\mathrm{TM}}$ system (Nalge Nunc International) chambers. The cells were transfected and remained in culture for 24-72 h, after which, they were fixed in $4 \%$ praraformaldehyde $(\mathrm{w} / \mathrm{v})$ for $7 \mathrm{~min}$ in room temperature (RT). Following washes in PBS, the cells were treated with PBS containing 10\% normal goat serum (NGS) (blocking buffer) for 1 hour in RT and incubated with the primary antibodies overnight $(\mathrm{O} / \mathrm{N})$ at $4^{\circ} \mathrm{C}$. The antibodies were diluted as follows in blocking buffer; chicken anti-GFP (1:500; Abcam: ab13970), rabbit anti- $\delta$-catenin (1:50; Santa Cruz Biotechnology Inc. (H-160) sc-33553), rabbit anti-SPHK1 antibody (1:100; Abcam: ab71700), rabbit anti-Pax6 (1:300; Covance: PRB-278P-100), rabbit anti-Ki67 (1:100; Vector Laboratories, Enzo Life Sciences GmbH, Lörrach, Germany VP-RM04), rabbit anti-phospho-Histone H3 (1:500; Abcam: ab5176), rabbit anti-PDGF BB (1:300; Abcam: ab23914). Primary antibodies were detected with appropriate secondary antibodies conjugated to Alexa Fluor fluorophores (1:500; Invitrogen), and cells were counterstained with Vectashield mounting medium containing 4',6'diamidino-2-phenylindole (DAPI) (Vector Laboratories, Enzo Life Sciences GmbH, Lörrach, Germany) to label the cell nuclei. Five randomly selected microscopic fields were selected and pictured for 
Table I. Primers used for PAX6-3'UTR mutagenesis.

\begin{tabular}{ll}
\hline Primers & \multicolumn{1}{c}{ Sequence } \\
\hline PAX6 mutant_A 3'UTR & Forward: ATTGTCTAGAGGAAAGGAAATATTGTGTTAGCTCAG \\
& Reverse: GCTTTCTAGATCATGACCAACACAGATCAAAC \\
PAX6 mutant_B 3'UTR & Forward: CAGGTCTCTAGACCATGTGAAAGCCTTTGTATTTCC \\
& Reverse: GTAACTTCTAGACTGACAATGGAAATCTGCCCAG
\end{tabular}

each condition and the positive cells counted. The images were acquired using an Olympus XC30 camera attached to an Olympus BX60 fluorescence microscope and they were processed with Microsoft's power point format-picture command for corrections in brightness/contrast.

Luciferase reporter assays. The 3'UTR of Pax6, was cloned into the pGL3-promoter reporter vector (Promega, $\mathrm{GmbH}$, Mannheim, Germany). Constructs carrying mutations in the potential targeting sites were also generated and used as controls. Transfections were performed in 24-well plates using Lipofectamine 2000 (Invitrogen Life Technologies $\mathrm{GmbH}$, Darmstadt, Germany) and the following vector DNA concentrations per well: 0.4ng Pax6 3'UTR construct (or Pax6 3'UTR-mutated construct or pGL3 empty plasmid), $0.4 \mathrm{ng}$ miRNA expression plasmid, 0.2 ng Renilla Luciferase plasmid (Promega, GmbH, Mannheim, Germany). Dual-Luciferase Reporter Assay (Promega, GmbH, Mannheim, Germany) was carried out $48 \mathrm{~h}$ after transfection and Firefly luciferase activity (F) was normalized to Renilla luciferase reporter activity (F/R). The value $(\mathrm{F} / \mathrm{R})$ of the miRNA expression plasmid + pGL3 empty vector transfected cells, was set to 1 .

Cell culture and transfections. U118, U87 and U251 glioma cell lines as well as HeLa and P19 cells were purchased from Cell Lines Services, and were grown in Dulbecco's modified Eagle's medium (DMEM) (Gibco), supplemented with $10 \%$ Fetal Bovine Serum (FBS) (Gibco). Transient transfections of cells were performed using Lipofectamine LTX with PLUS reagent (Invitrogen) and endotoxin-free DNA plasmids (Qiagen kit) or commercially purchased (Qiagen) miRNA mimics and inhibitors (see miRNA expression vectors, mimics and inhibitors section of Materials and methods), in 96-well plates, 24-well plates and Lab-Tek II Chamber Slide system chanbers (Nalge Nunc International) according to the supplier's guidelines.

Human glioma samples. The collection of the glioma tumor specimens was done in collaboration with the Medical University, Varna, Bulgaria. (Department of Anatomy, Histology and Embryology - Head, Professor A. Tonchev; The Neurosurgery Clinic - Director, Dr Y. Enchev; Department of Neurology - Dr A. Kaprelyan). In total 22 GBM and 7 lowgrade glioma tumors were analyzed in Max Planck Institute for Biophysical Chemistry, Göttingen, Germany. The RNA extraction and qRT-PCR analyses were done as described in previous sections. The collection and use of tissues were approved by the Ethics Committee of the Medical University of Varna.
Cell culture assays. To perform wound-healing assay, U87MG cells were seeded in 24-well plates and transfected with miRNA expression plasmids, miRNA inhibitors, control GFP-expressing plasmid, PAX6 or PAX6-5a expression plasmids. When the cells reached confluence, a wound was gently made through the central axis of the plate, by scraping the cell monolayer with a micropipette $200 \mu \mathrm{l}$ tip. The cultures were washed with serum free (SF) media to remove floating cells and placed back in the incubator. Pictures of the scratches were taken at selected time points, in five randomly selected microscopic fields for each condition and time point, using a digital camera Prog/Res/3012 from Kotron Elektronik coupled to a Leitz Labovert microscope (Leica). The images were processed with Microsoft power point format-picture command, for brightness and contrast corrections. The same filter was applied in every picture as to make the cells clearer and to correct any background coloration.

The invasion assays were performed using the BD Biocoat $^{\mathrm{TM}}$ kit (BD Matrigel Invasion Chamber 24-well plate 8.0 Micron; BD Biosciences; Heidelberg, Germany) as instructed by the supplier. The invaded cells on the lower membrane surface were fixed in $100 \%$ methanol for $10 \mathrm{~min}$, air-dried, stained with DAPI and counted under an Olympus BX60 fluorescence microscope. In the cell survival assays U87 cells were seeded in 24-well plates and allowed to reach $80 \%$ confluence in media with serum, followed by washes in SF-media. The cells were then transfected with either miRNA or PAX6/PAX6-5a expression plasmids and allowed to grow in SF-media for $72 \mathrm{~h}$ in total. Pictures were taken at defined time points with a digital camera Prog/Res/3012 from Kotron Elektronik coupled to a Leitz Labovert microscope (Leica).

Western blot (WB) analysis. WB analyses were done as previously described (36). The following primary and secondary antibody dilutions were used: rabbit anti-Pax6 polyclonal antibody (1:500; Covence: PRB-278P-100), rabbit anti- $\delta$-catenin (1:200; Santa Cruz Biotechnology Inc.: (H-160) sc-33553), rabbit anti-PDGF BB (1:500; Abcam: ab23914), rabbit anti$\beta$-actin polyclonal antibody (1:500, Abcam: ab8227). $\beta$-actin served as loading control and PageRuler pre-stained protein ladders (Fermentas) were utilized for the identification of the correct sized proteins.

Caspase 3/7 assays. Cell death measurement studies were performed using a Caspase-Glo ${ }^{\circledR}$ 3/7 Assay System from Promega, according to the manufacturer's instructions. The luminescence was measured in a Centro LB 960 Luminometer (Berthold Technologies). 
Ago2 immunoprecipitation of targets. The Ago2 IP experiments were done exactly as previously described (30), using P19 cells seeded in 24-well plates. The immunoprecipitated RNA was analyzed by qRT-PCR using gene-specific primers and normalized to U6 RNA. GAPDH served as negative control. The analysis was done as follows: GFP control vector, pull-down/input (A); miRNA, pull-down/input (B); fold enrichment $=\mathrm{B} / \mathrm{A}$.

Algorithm tools/Target prediction databases. The following algorithms were applied to find miRNAs that target PAX6: Targetscan (http://www.targetscan.org), DIANAmT (http:// diana.imis.athena-innovation.gr/DianaTools/index. php?r=microT_CDS/index), miRWalk (http://www.umm.uniheidelberg.de/apps/zmf/mirwalk/), PICTAR 5 (http://pictar. mdc-berlin.de), RNA22 (http://cbcsrv.watson.ibm.com/rna22. html). For the analysis of the miR-183-96-182 promoter for putative PAX6 binding sites, we used the RVISTA 2.0 database (http://rvista.dcode.org).

\section{Results}

Upregulation of the miR-183-96-182 cluster correlates with reduced level of PAX6 and PAX6-5a and increased glioma progression. Although high expression of $P A X 6$ has been assumed to slow down the GBM progression (20), the specific role of each of the $P A X 6$ isoforms has not been approached so far. In order to determine whether the expression of PAX6/PAX6-5a and miR-183-96-182 cluster correlates at different stages of glioma progression, we utilized qRT-PCR ( $\Delta \Delta \mathrm{Ct}$ relative quantification) to analyze primary human glioma tissue specimens (29 samples in total) and glioma cell lines (U118, U251 and U87). Fig. 1A shows the relative expression levels of both PAX6 isoforms that are significantly lower (7.712.5-fold down, respectively) in GBM (22 samples of primary GBM, grade IV) compared to astrocytomas (grades I and II; 7 samples in total). In the tested four high-grade glioma cell lines, PAX6/PAX6-5a level was markedly downregulated compared to low-grade glioma tumors. On the contrary, all members of the miR-183-96-182 cluster were highly upregulated in GBM, as well as in high-grade glioma cell lines (Fig. 1B). miR-96 appears the most abundantly expressed miRNA in glioblastoma tumors, showing more than 14-fold upregulation compared to low-grade astrocytoma (Fig. 1B).

PAX6 is a direct target of miR-183-96-182 cluster. These observations prompted us to inquire more into the possible regulatory relationship between the PAX6 isoforms and the microRNA cluster. Using several target prediction programs (see Materials and methods) we examined whether the 3'-UTR of PAX6 contains putative sites for miR-183, miR-96 and miR-182 interactions. All programs predict one binding site for each miR-182 and miR-96 in the 3'UTR region of the PAX6 gene. Both sites, are eptamers (7mer-1A) and similar for the two miRNAs because their seed sequences are alike (Fig. 1C). MiR-183 complementarity is predicted by two algorithms (miRanda and miRWalk).

To validate direct binding and targeting by miR-18396-182, we constructed firefly luciferase reporter plasmids containing either wild-type or mutated 3'UTR of PAX6. In order to disrupt the predicted binding, plasmids containing mutations within the miR-183, miR-96 and miR-182 seedbinding regions in the 3'UTR of PAX6 were designed and generated. Reporter activities were quantified and normalized to non-targeted Renilla luciferase activity. As shown in Fig. 1D, cotransfections of miRNA expression plasmids together with wild-type PAX6-3'UTR-reporter in HeLa cells, resulted in pronounce reduction of the relative luciferase activity, approximately 2.9 -fold by either miR-182 or miR-183 and 2.7-fold by miR-96. Mutations (Table I) of the predicted specific miRNA recognition elements completely restored luciferase expression (Fig. 1D). To further confirm the direct regulation of $P A X 6$ and $P A X 6-5 a$ by miR-182-96-183 cluster, we utilized AGO2 immunoprecipitation of targets (AGO2-IP) as previously described (30). The analysis of the results shows that miR-182/AGO2, miR-183/AGO2 and miR-96/AGO2 complexes associate selectively with the $P A X 6$ or $P A X 6-5 a$ transcripts (Fig. 1E). The levels of both PAX6 and PAX6-5a mRNA were significantly higher (more than 2.5-fold) than control transcripts (U6 RNA and GAPDH mRNA) (Fig. 1E), suggesting that both PAX6 and PAX6-5a are targeted by the clustered miRNAs.

Frequently, miRNAs and TFs participate in feed-forward and feedback loop regulatory networks $(37,38)$. A search for putative binding sites of PAX6 in the promoter of miR-18396-182 in silico, identifies TFs PAX6 and BACH2 as candidate regulators of the miR-183-96-182 cluster (based on highly conserved sequences between species and known TF binding motifs within these regions, the RVISTA database) (data not shown).

miR-183 preferentiallyregulates $P A X 6-5$ a expression in glioma cells. To further investigate whether PAX6 and PAX6-5a are specifically targeted by the miRNA cluster we used qRT-PCR and WB, to compare $P A X 6 / P A X 6-5 a$ expression in glioma cell lines (U87, U118, U251) transfected with microRNA mimics or inhibitors. All glioma cell lines used have, minimal levels of both PAX6 and PAX6-5a (Fig. 1A). Since the results were similar for all cell lines, only those obtained from U87 cells are shown, unless otherwise specified.

Noteworthy, inhibition of miR-183 alone results in significant increase of the PAX6-5a protein levels while PAX6 is only insignificantly affected (Fig. 2A2). On the other hand, inhibition of either miR-182 or miR-96 affects the expression of both PAX6 and PAX6-5a. Noteworthy, the regulatory relationship between the two PAX6 isoforms is by itself complex. Despite slight fluctuations during embryogenesis, under physiological conditions, PAX6 is maintained at higher levels than PAX6-5a. In addition, PAX6 directly and positively autoregulates itself and PAX6-5a, while PAX6-5a regulates PAX6 expression in a dose-dependent manner (39-41). Accordingly, increase of PAX6 levels would also result in an increase of the PAX65a, while higher PAX6-5a, as is the case when miR-183 is inhibited (Fig. 2A2), results in decrease or no-change of the PAX6 levels. However, inhibition of the whole miRNA cluster leads to an increase of both PAX6 and PAX6-5a protein levels (Fig. 2A3).

Consistently, after overexpression (OE) of miR-182, miR-96, or the whole miRNA cluster, the level of PAX6 is reduced (Fig. 2A4, A5 and A6), while miR-183 OE does not 


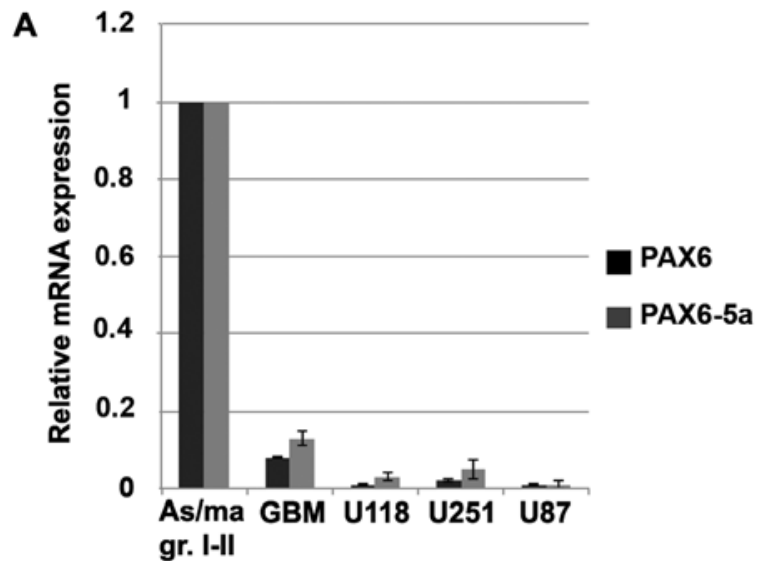

C

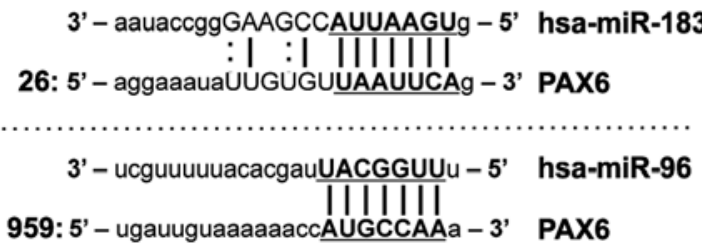

3' - ucacacucaagaugguUACGGUUU - 5' hsa-miR-182 IIIIII

959: 5' - uugauuguaaaaaaccAUGCCAAa - 3' PAX6

E

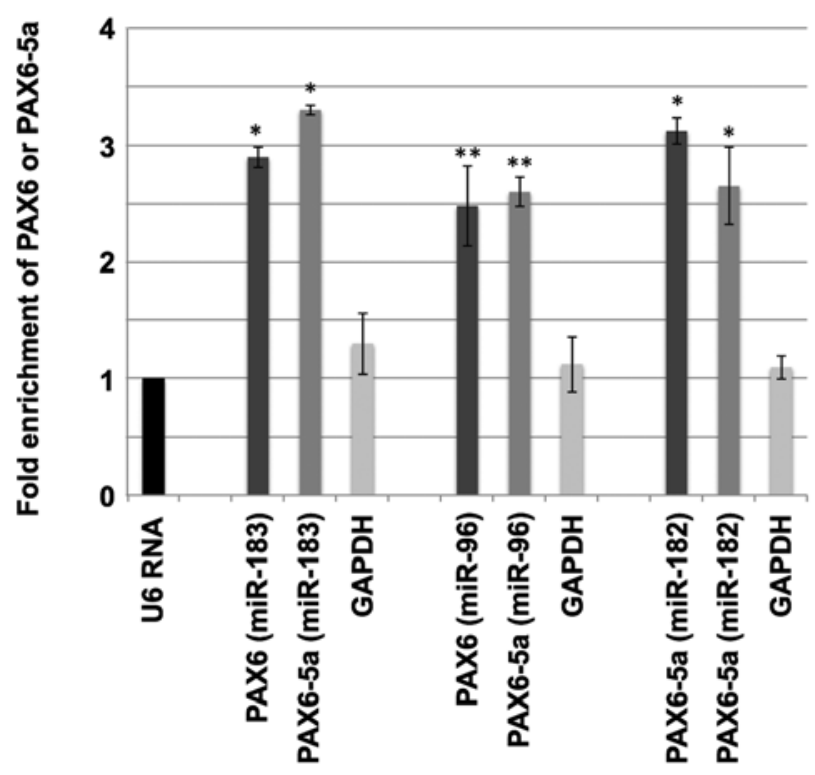

B

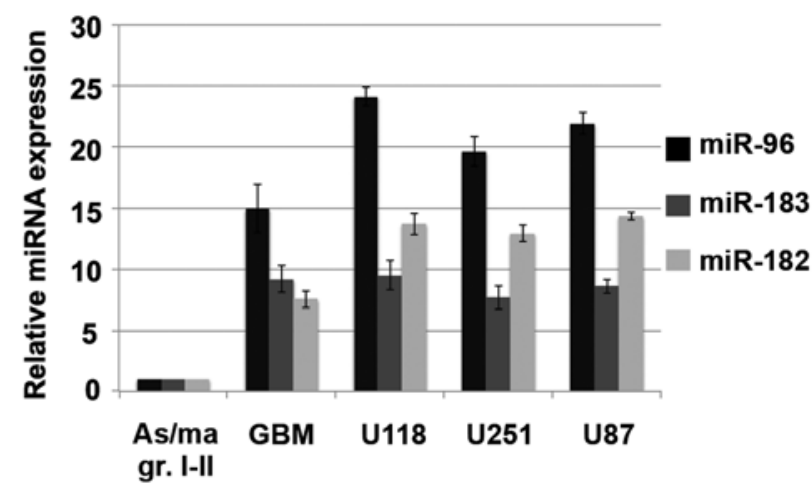

D

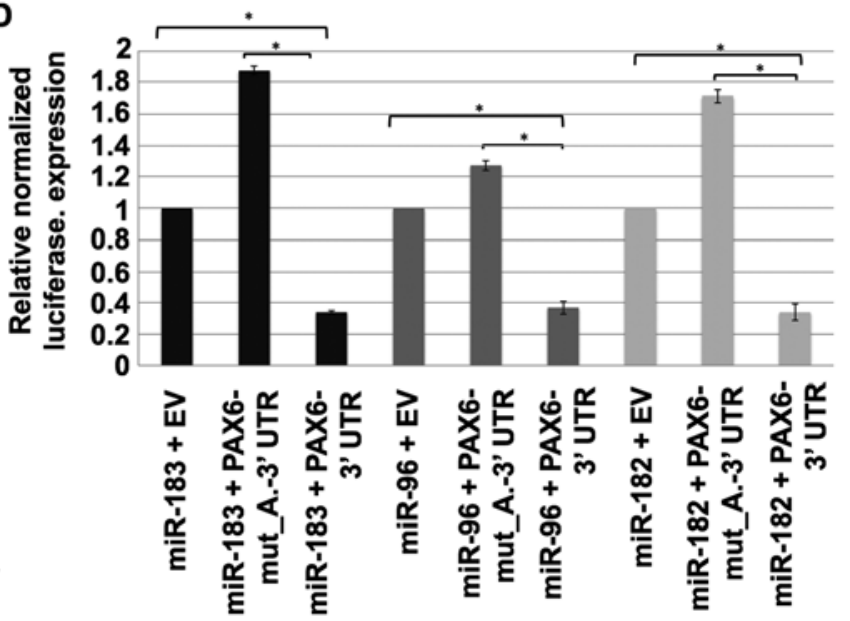

Figure 1. Reciprocal interaction between members of miR-183-96-182 cluster and PAX6/PAX-5a, showing opposite expression pattern in glioma. (A) The expression of both PAX6 and PAX6-5a isoforms is severely repressed in GBM (grade IV astrocytoma) and in high-grade glioma cell lines (U118, U251, U87). Error bars show SEM from: 22 GBM samples and 7 low-garde glioma samples (As/ma gr. I-II: Astrocytoma tumors grades I-II) for the tumor biopsies and 3-4 independent experiments for the cell lines; $\mathrm{P}<0.05$ for all glioma lines and cell lines. (B) The opposite expression pattern is seen for miR-183-96-182 cluster. Error bars show SEM from: 22 GBM samples and 7 low-garde glioma samples (As/ma gr. I-II: Astrocytoma tumor grades I-II) for the tumor biopsies and 3-4 independent experiments for the cell lines; $\mathrm{P}<0.05$ for all glioma lines and cell lines. (C) The seed sequences of miR-182 and miR-96 are similar, thus binding to the same region on the PAX6 3'-UTR. (D) Each member of the miR-183-96-182 cluster directly targets the PAX6 3'-UTR. HeLa cells were cotransfected with either miR-183, miR-96, miR-182 expression vectors and luciferase reporters containing either the predicted miRNA target sites in the PAX6 3'UTR, or a mutated form (Mut.), which was used as a positive control ("P $<0.01$; Error bars represent SEM from 4 independent transfections; EV: empty vector). (E) Both PAX6 and PAX6-5a isoforms interact with the three cluster members. HeLa cells were cotransfected with expression vectors for HA-tagged AGO2 and miR-183, miR-96 and miR-182. The immunoprecipitated RNA was analyzed by qRT-PCR using specific primers either for both PAX6 and PAX6-5a or for PAX6-5a alone and normalized to U6 RNA. GAPDH served as negative control. Both PAX6 and PAX6-5a were significantly enriched in the pull-downs $\left({ }^{*} \mathrm{P}<0.01 ;{ }^{* *} \mathrm{P}<0.05\right.$; Error bars represent SEM from 4 independent transfections).

seem to significantly affect PAX6 level (Fig. 2A6). Notably, in both cases, PAX6-5a, which is expressed at lower levels in the native U87 cell line, is not detectable. Similar results were obtained at the mRNA level, where knockdown (KD) of any of 
A

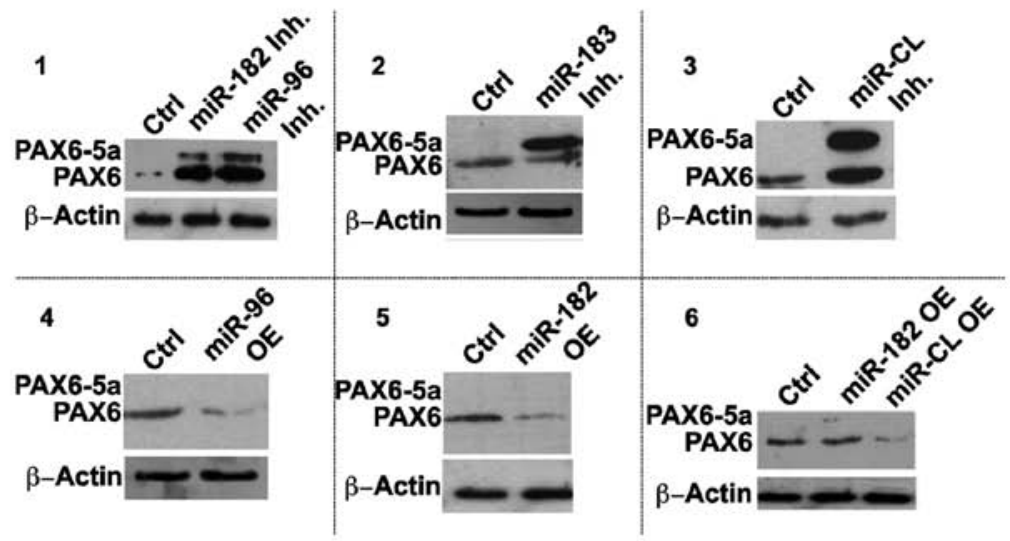

B

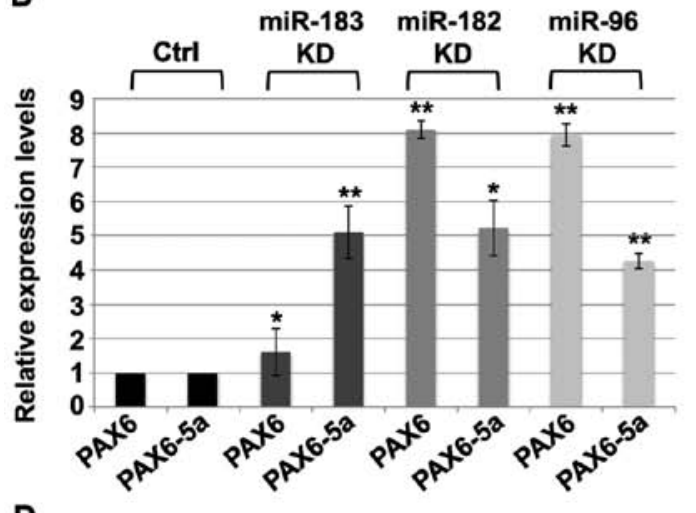

D

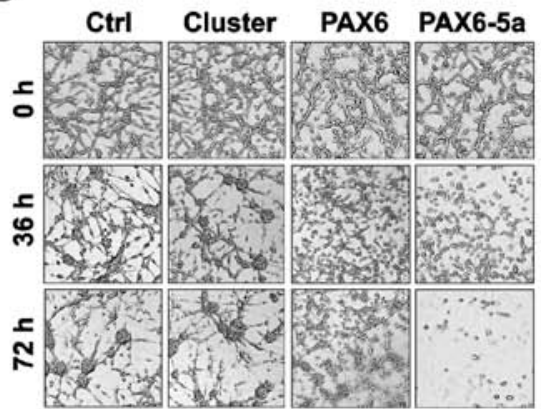

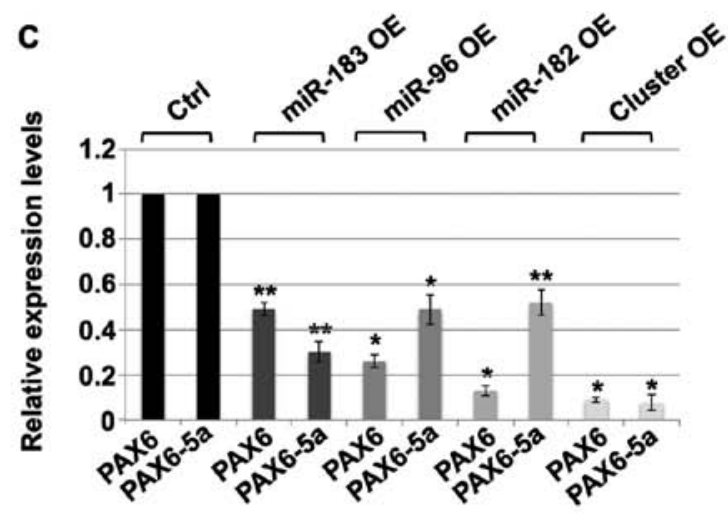

$\mathbf{E}$

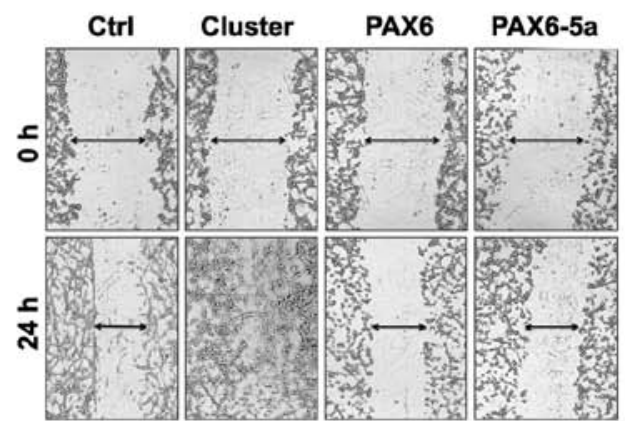

$\mathbf{F}$

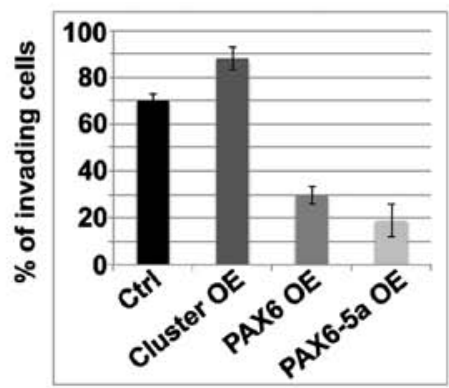

Figure 2. miR-183-96-182 cluster negatively regulates the expression of both PAX6 and PAX6-5a isoforms and affects tumorigenicity of glioma cell lines. (A) Western blotting analysis of the PAX6 and PAX6-5a protein levels in glioma cell line U87MG, after inhibition (Inh.) or overexpression (OE) of single components of the miR-183-96-182 cluster or the whole cluster (miR-CL). (B) qRT-PCR analysis of PAX6 and PAX6-5a mRNA, in U87 cells, after miR-183, miR-96 and miR-182 knockdown (KD) using specific miRNA inhibitors (details are shown in Materials and methods) ("P<0.01; ** P<0.05; Error bars show SEM from 4 independent transfections) or (C) after OE of miR-183, miR-96, miR-182 or the cluster $\left({ }^{*} \mathrm{P}<0.01\right.$; ${ }^{* *} \mathrm{P}<0.05$; Error bars show SEM from 4 independent transfections). (D) Time course (0-72 h) of U87MG cell survival potential following simultaneous transfection with miR-182 miR-96 and miR-183 (Cluster), PAX6 and PAX6-5a. Note that cells transfected with the miRNAs (cluster) proliferate faster and start to form large clusters, similarly to the control native cells, while the cells transfected with either PAX6 isoform fail to form clusters and become apoptotic. The latter is especially prominent after transfection with the PAX6-5a expression plasmid (representative images from 1 out of 3 independent experiments). (E) Time course (0-24 h) of U87MG glioma cell migration in a monolayer-based wound-healing assay (scratching assay), after OE of the miR-183-96-182 cluster (Cluster), PAX6 and PAX6-5a. Transfection of either PAX6 or PAX6-5a markedly reduces the migratory potential of the glioma cells, while the opposite is evident after miRNA cluster OE. The gap is indicated by double arrows. (representative images from 1 out of 3 independent experiments). (F) The PAX6 or PAX6-5a OE U87MG cells become less invasive compared to high miR-183-96-182 cluster expression conditions, as indicated by Matrigel invasion assays (details in Materials and methods). (Cluster: miR-182 mimic, miR-96 mimic and miR-183 mimic simultaneous transfection. Control: non-transfected U87MG cells. Values represent average percentage of invading cells from 3 independent experiments. Error bars show SEM. P<0.05). 
the miR-183-96-182 members, results in increased expression levels of both PAX6 and PAX6-5a (Fig. 2B), while the opposite is obvious following OE of any of the miRNAs (Fig. 2C). At the mRNA level it is also evident that miR-183 has a greater impact on the expression levels of PAX6-5a, while PAX6 is only mildly increased (Fig. 2B and C). On the contrary, miR-182 and miR-96, both have more influence on the expression levels of the PAX6 isoform (Fig. 2B and C).

Overexpression of miR-183-96-182 promotes glioma cell migration, survival and proliferation in glioma cell lines. Next, we transfected glioma cell lines U87, U118, U251 with either mimics specific for each component of the cluster, or with PAX6 and PAX6-5a expression constructs and examined the effect on the survival and migration of the cells. Since the results were similar for all cell lines, only those obtained from U87 cells are shown, unless otherwise specified. First, we used a simple cell viability assay to assess the effect the whole miRNA cluster on the survival of U87 cells, compared to PAX6 or PAX6-5a OE. As shown in Fig. 2D the miRNA cluster promoted the survival of glioma cells, indicated by the formation of large clumps from $36 \mathrm{~h}$ onwards. On the contrary, PAX6 OE caused decreased cell viability, while the effect of the OE of PAX6-5a isoform was even more prominent (Fig. 2D).

The migratory potential of glioma cells, was further evaluated by wound healing (scratch) assays after OE of the whole miRNA cluster. PAX6 and PAX6-5a OE markedly slowed down the migration. This is evident by the closure of the wound only by approximately $30 \%$ in $24 \mathrm{~h}$, closely resembling the control situation. In the latter, the closure was more prominent $(38 \%)$ with few cells already migrated in the middle of the wound (Fig. 2E). In order to circumvent the assumed cell migration that might partially be the result of cell proliferation, we limited the experiment to $24 \mathrm{~h}$. Nevertheless, further future experiments are required in order to assess absolute rates of total cell proliferation and determine net migration independent of proliferation.

Lastly, we examined the invasive ability of glioma cells by applying matrigel transwell assays. We found that upon miRcluster OE, the cells become more invasive (more than $80 \%$ of the transfected cells have migrated through the matrigel), whilst upon PAX6 or PAX6-5a OE, $30 \%$ and $24 \%$ of the cells invaded, respectively (Fig. 2F). Overall, these findings indicate that U87 glioma cells acquire higher tumorigenic aptitude after transfection with miR-183, miR-96 and miR-182 mimic oligonucleotides, whereas PAX6 and PAX6-5a push them towards a less motile, non-invasive and non-viable track.

In order to evaluate whether the restrained survival, migration and invasion of the glioma cells after PAX6/PAX6-5a OE is due to aberrant proliferation, we performed immunostainings with Ki67, which marks cells at any stage of proliferation and $\mathrm{pHH} 3$, as a specific mitotic marker, using U87 cells. Our analysis shows a reduced number of $\mathrm{Ki} 67$ and $\mathrm{pHH} 3$ positive cells after transfection with PAX6 OE $\left(3.4 \pm 1.55 \% \mathrm{Ki}^{+}\right.$cells; $1.4 \pm 0.23 \% \mathrm{pHH}^{+}$cells) or PAX6-5a OE constructs $\left(2.34 \pm 1.77 \% \mathrm{KI}^{+} 7^{+} ; 2.5 \pm 0.98 \% \mathrm{pHH}^{+}\right.$cells) (Fig. 3). On the contrary, transfection with mimics for miR-183 or miR-182/96 results in significantly increased proliferation rates (miR-183 transfection: $98.7 \pm 3.66 \% \mathrm{Ki}^{+}$and $97.5 \pm 2.14 \% \mathrm{pHH}^{+}$cells;
miR-182/96 transfection: $92.6 \pm 2.75 \% \mathrm{Ki}^{+}$and $92.5 \pm 5.67 \%$ pHH3 ${ }^{+}$cells); control GFP transfection: $93.7 \pm 6.88 \% \mathrm{Ki}^{+}{ }^{+}$and $94.78 \pm 3.66 \% \mathrm{pHH}^{+}$cells) (Fig. 3).

Finally, we examined whether the reduction of U87 cell proliferation rate after PAX6/PAX6-5a OE is also associated with increased cell death. To test this, we utilized a generic enzymatic assay that measures caspase- 3 and -7 activity. After OE of PAX6 or PAX6-5a, we observed a 1.9- and 2.2-fold activation of these effector caspases, respectively. At the same time, inhibition of the miR-cluster resulted in 2-fold higher enzyme activity, compared to the controls. These findings suggest that miR-183-96-182 regulate glioma growth and proliferation by inhibiting PAX6 and PAX6-5a, which otherwise, would make the cells less viable.

PAX6 and PAX6-5a specifically regulate a number genes involved in GBM progression. From our literature search we assorted at least 8 putative candidates [(SPHK1, CTNND2 ( $\delta$-catenin), PDGF-A, PDGF-B, PDGFR- $\alpha, P D G F R-\beta$, Neurofibromatosis $1(N F 1)$, and T-cell lymphoma invasion and metastasis 1 (TIAM1)] that could be downstream known or putative interaction partners of PAX6 and PAX6-5a during glioma progression. Using qRT-PCRs assays and $\Delta \Delta \mathrm{Ct}$ quantification, we found that the expression of $P A X 6$ and $P A X 6-5 \mathrm{a}$ (Fig. 4A) is highly downregulated in 22 high-grade glioma tumors, while at the same time the expression of SPHKI is upregulated by $3.27 \pm 0.049$. SPHK1, an oncogenic enzyme known to contribute to the acquisition of the apoptosis resistance of the glioma cells, has recently been suggested as a direct downstream target of PAX6-5a (42-44), but such an interaction has not been validated so far. On the other hand, the central nervous system specific protein CTNND2 is known to be regulated by PAX6 and PAX6-5a, in a dose- and isoform-sensitive fashion (45). Of note, high levels of CTNND2 correlate with positive patient outcome, while inactivation of CTNND2 is a key genetic alteration driving the aggressive mesenchymal phenotype of GBM (46). In agreement with previous data (45), in our 22 GBM samples we observed a decreased expression of CTNND2 accompanied by a low PAX6 and PAX6-5a expression level. Finally, we found that the relative expression of $P D G F-B$ and the corresponding receptor $P D G F R-\beta$ was highly diminished by $9.09 \pm 0.00154$ and $4.34 \pm 0.0038$-fold, respectively, during glioma progression.

Intrigued to further explore the connection of $P A X 6$ isoforms with $S P H K 1$, and $P D G F-B$, we constructed firefly luciferase reporters containing the putative promoter region of $S P H K 1$ and $P D G F-B$ and we co-transfected each construct with either $P A X 6$ or $P A X 6-5$ a OE plasmids in HeLa cells. Transfections with either PAX6 + pGL3-luciferase or PAX6-5a + pGL3-luciferase empty vector were used as control. The luciferase activity was reduced by $0.56 \pm 0.096$-fold when PAX6 was co-transfected with SPHK1/PGL3-Luciferase construct and 0.46 \pm 0.0225 -fold upon PAX6-5a co-transfection (Fig. 4B) suggesting that PAX6-5a tends to exert a slightly stronger effect. Co-transfection of PDGF-B/PGL3-Luciferase construct with PAX6 resulted in minor but not significant upregulation $(1.46 \pm 0.112$-fold) of the luciferase activity. However, when co-transfected with PAX6-5a, the luciferase reporter activity was upregulated by more than $3 \pm 0.089$-fold, suggesting a specific positive regulation of PDGF-B by the 
A

PAX6
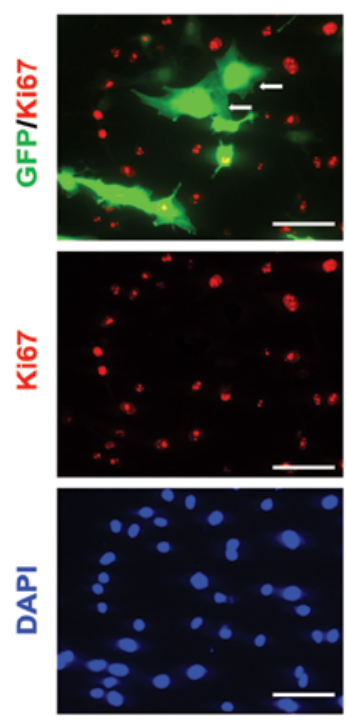

B
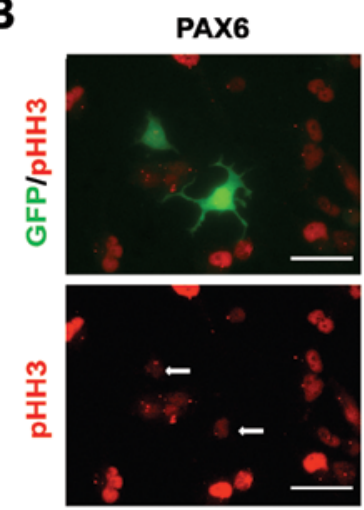

C

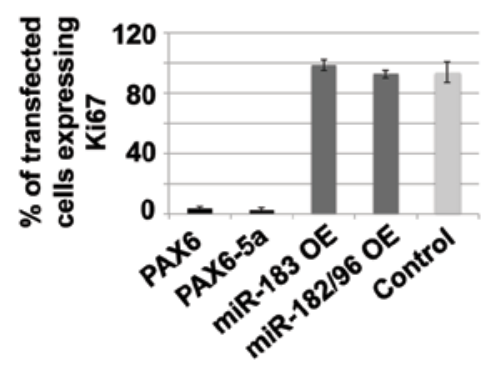

PAX6-5a
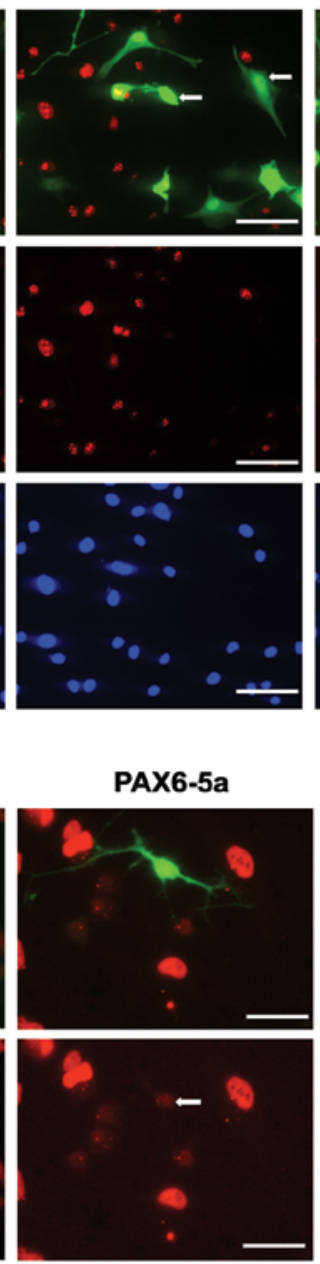

D

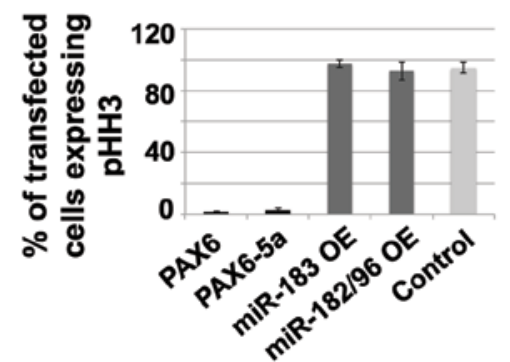

miR-183 OE
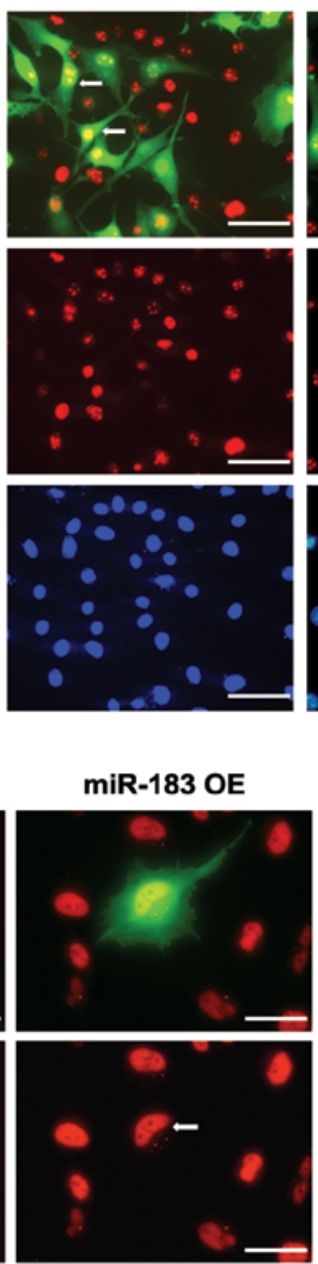

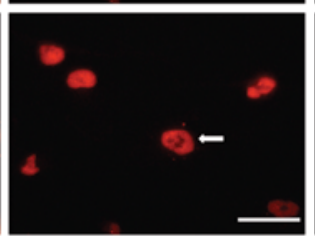

E

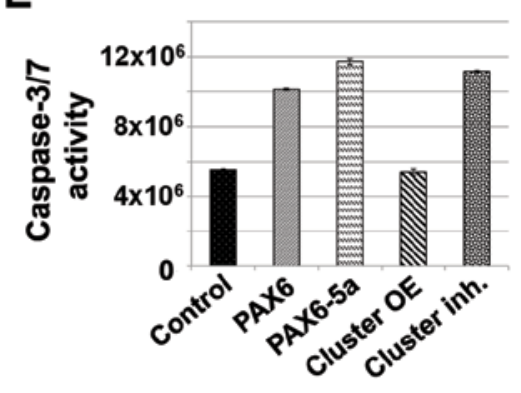

Figure 3. Assessment of proliferation and apoptosis of U87MG cells. (A) Immunofluorescence images of U87MG glioma cells transfected with PAX6-GFP, PAX6-5a-GFP, miR-183-GFP, miR-182/96-GFP and pCIG2-GFP control plasmids. Images show diminished proliferation after PAX6/PAX6-5a OE evident by the fewer $\mathrm{GFP}^{+} / \mathrm{Ki} 67^{+}$cells in (A); and diminished number of Ki67 $7^{+}$cells in $(\mathrm{C})(\mathrm{P}<0.001$; Error bars show SEM from 3 independent transfections). (B) Similarly, PAX6 and PAX6-5a expressing glioma cells, show diminished expression of the mitotic marker pHH3 (B and D) (P<0.001; Error bars show SEM from 3 independent transfections), suggesting that these cells have ceased to proliferate and may be dying, which is confirmed after measurement of caspase 3/7 activity (E) indicating increased cell death under PAX6/PAX6-5a OE conditions (in E; Values shown are from 1 representative experiment out of 3; Cluster OE: miRNA-183-96-182 cluster overexpression; Cluster Inh.: miR-183-96-182 cluster inhibition). (Scale bars: (A) $140 \mu \mathrm{m}$; (B) $100 \mu \mathrm{m}$ ).

PAX6-5a isoform. Together, all the above findings show that PAX6 and PAX6-5a could possibly regulate at least 3 key downstream effectors with important roles in glioma progression. However, additional ChIP experiments are required in order to absolutely verify this.

Specific regulation of PDGF-B, SPHK1 and CTNND2 by different PAX6 isoforms in glioma cell lines. We used glioma cells lines (U87, U118 and U251) in order to determine how PAX6 and PAX6-5a regulate PDGF-B, SPHK1 and CTNND2. Since the results were similar for all cell lines, only those obtained from U87 cells are shown, unless otherwise specified.

We overexpressed either PAX6 or PAX6-5a using specific for each isoform constructs, in U87MG cells (similar results were also obtained from U251 and U118 cell lines - data not shown). As shown in Fig. 5A and B, OE of PAX6 results in 
A

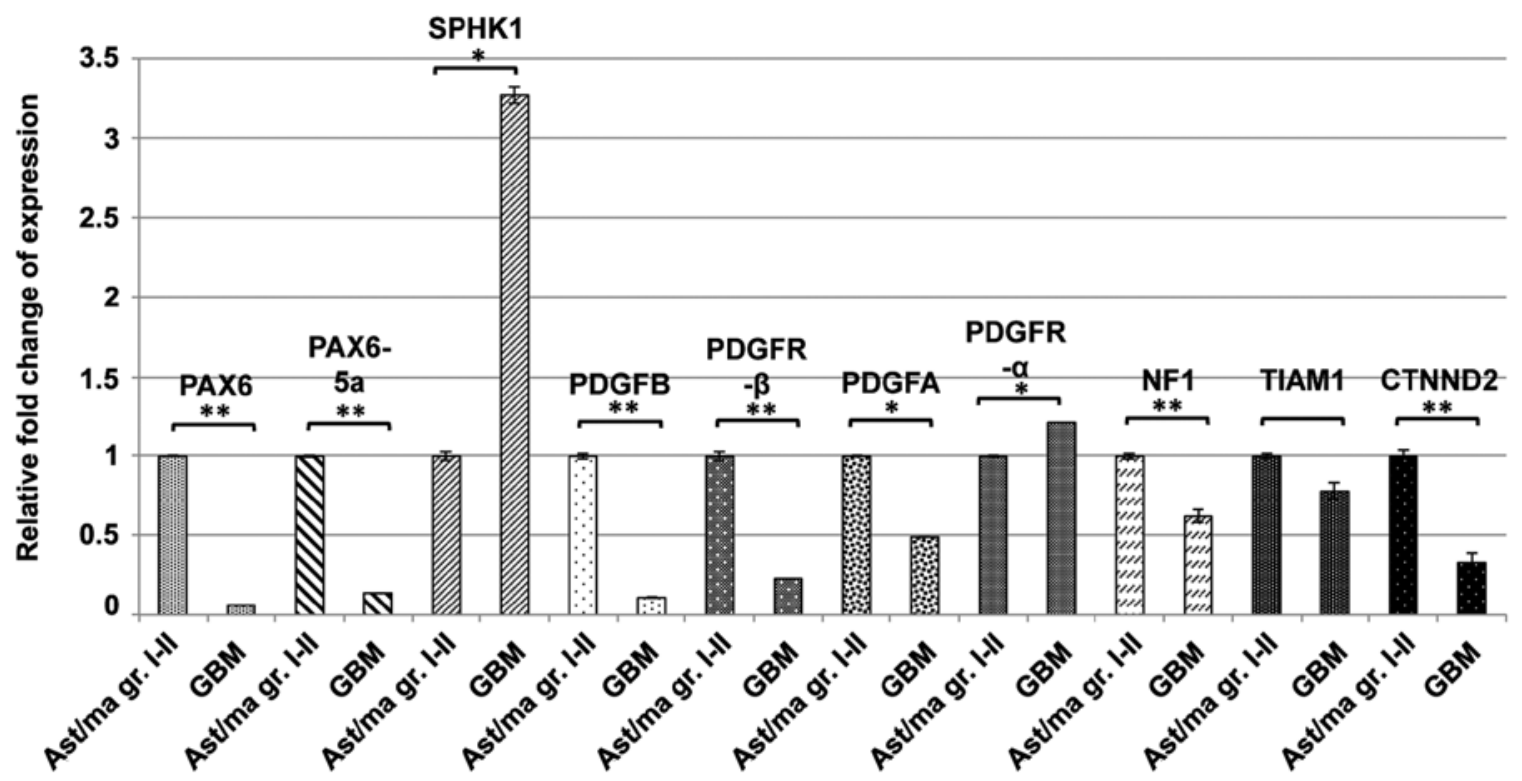

B

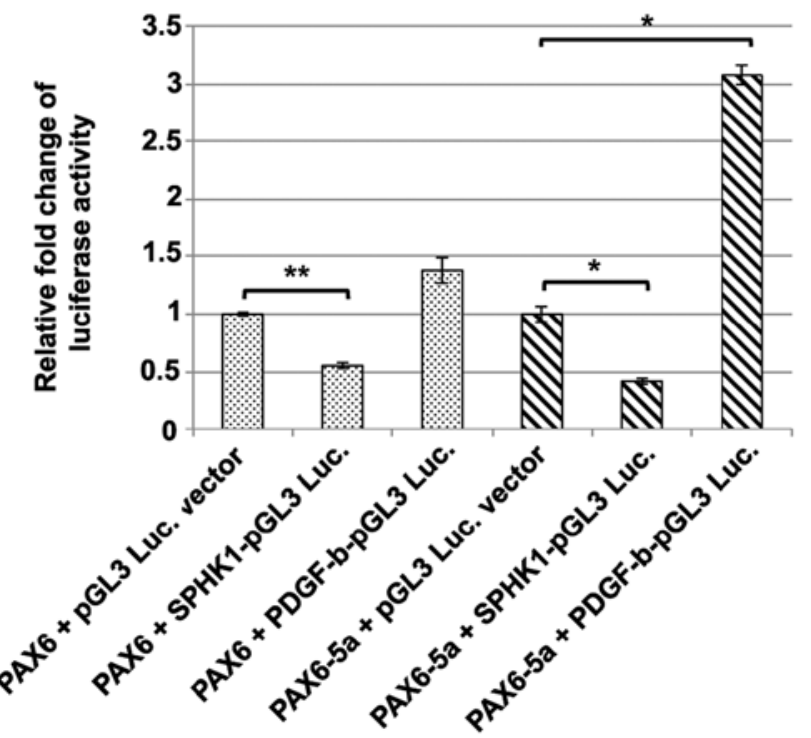

Figure 4. Deregulation of putative PAX6/PAX6-5a target genes in GBM in vivo. (A) Comparative qRT-PCR analysis of the expression levels of PAX6, PAX6-5a, SPHK1, PDGF-B, PDGFR- $\beta$, PDGF-A, PDGFR- $\alpha$, NF1, TIAM1 and CTNND2, from biopsies of diffuse fibrillary astrocytoma (stages I-II; 7 samples) and GBM (stages III-IV; 22 samples). The analysis indicates significant $\left.{ }^{* *} \mathrm{P}<0.05\right)$ downregulation of PAX6, PAX6-5a, PDGF-B, PDGFR- $\beta$ and $C T N N D 2$, while SPHK1 shows significant ("P<0.01) upregulation. PDGF-A, PDGFR- $\alpha$, NF1 and TIAM1 did not show any significant changes in this pool of samples. (Error bars show SEM from 22 GBM samples and 7 low-grade glioma samples). (B) Investigation of possible binding of PAX6 or PAX6-5a on the SPHK1 and PDGF-B promoters with luciferase reporter assays in NIH 3T3 cells indicates that SPHK1 is negatively regulated by both PAX6 isoforms although more prominently by PAX6-5a ( $\left.{ }^{*} \mathrm{P}<0.01 ;{ }^{* *} \mathrm{P}<0.05\right)$. On the contrary, PDGF-B is positively regulated exclusively by PAX6-5a, while PAX6 exerts only a minor effect $($ P $<0.01)$ (Error bars show SEM from 4 independent transfections).

minor (2.12 \pm 0.39 -fold) upregulation of PDGF-B, whereas PAX6-5a OE yields a much stronger effect, causing a $7.59 \pm 0.52$-fold upregulation of the PDGF-B levels. Although previously we were able to show (Fig. 4B) that PAX6-5a and not PAX6 is the actual regulator of PDGF-B, it is expected that PAX6 OE would result in a similar, but milder to PAX6-5a OE effect, due to the reported regulation of the PAX6-5a by PAX6 $(44,45,75)$. In our experiments we also observed upregulation (4.6 \pm 0.54 -fold) of PAX6-5a under the PAX6 OE conditions, explaining the slight upregulation of PDGF-B by
PAX6 OE. The effect, naturally becomes more prominent after higher (10.6 \pm 0.64 -fold) PAX6-5a OE as seen in Fig. 5B. On the other hand, we did not witness 'strong' upregulation of PAX6 after PAX6-5a OE, meaning that PAX6 probably regulates PAX6-5a but not necessarily vice versa, or that the PAX6-5a isoform regulates negatively PAX6 after a certain OE level. The latter has been previously reported (45). Our WB analysis shown in Fig. 5C is in accordance with the above.

We examined the expression of SPHKl level, after OE of $P A X 6$ or $P A X 6-5 a$ and we found a 2.4- and 7.7-fold reduction 
A

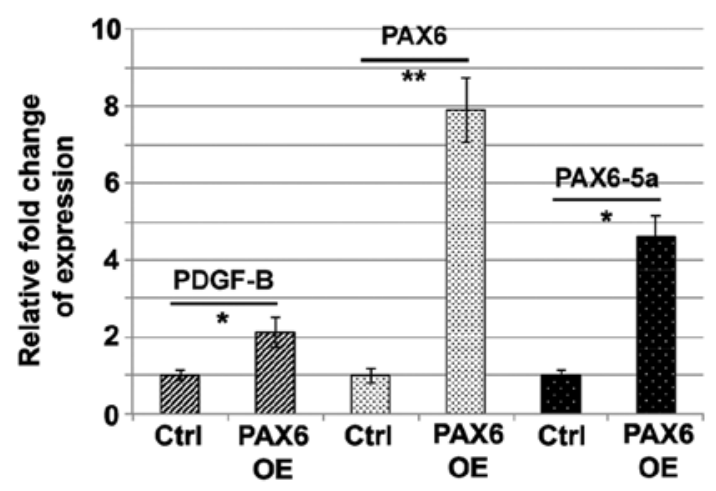

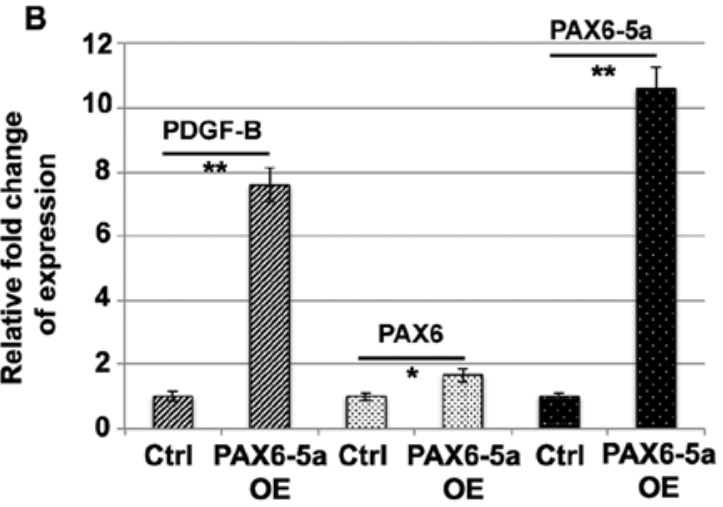

E

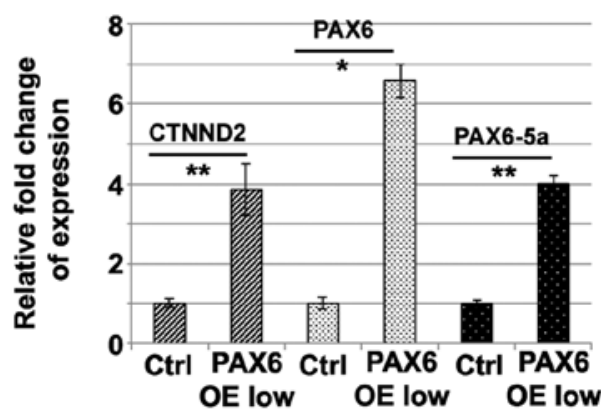

$\mathbf{F}$

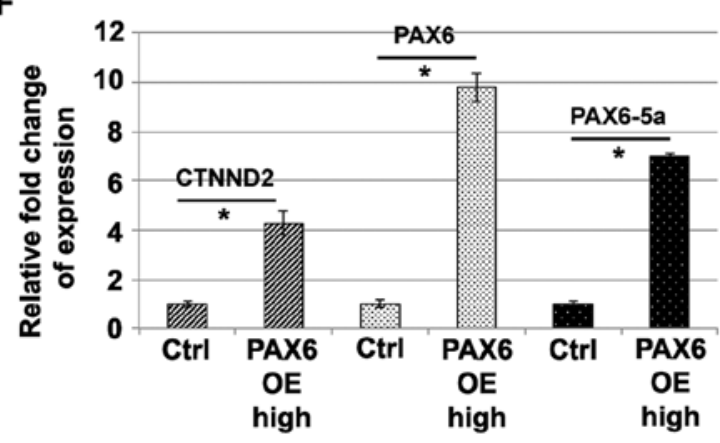

H

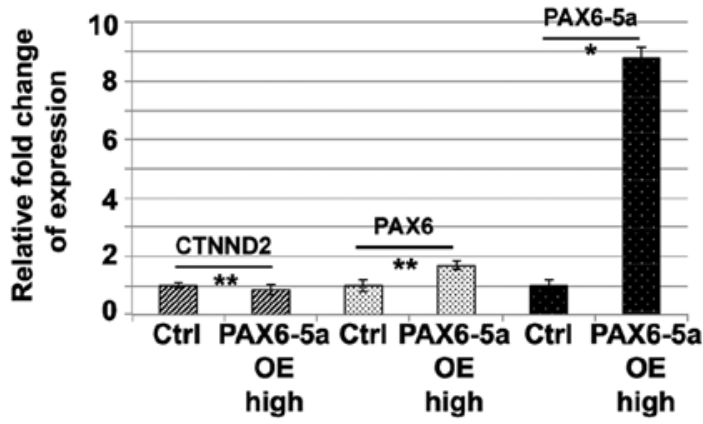

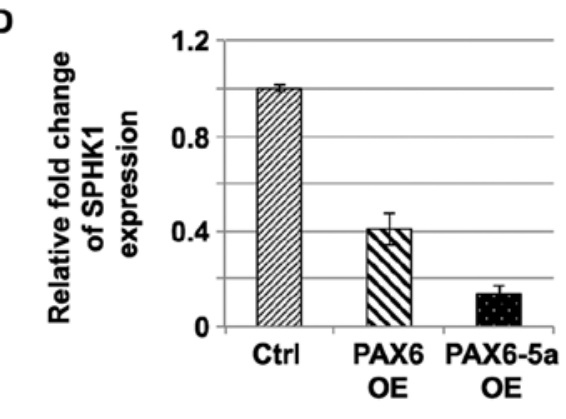

G

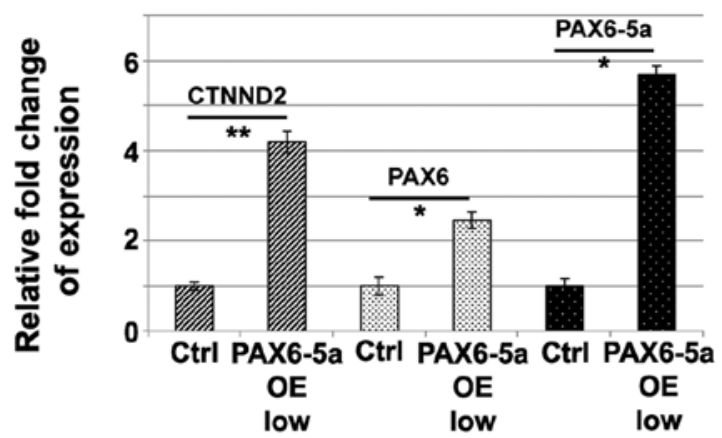

I

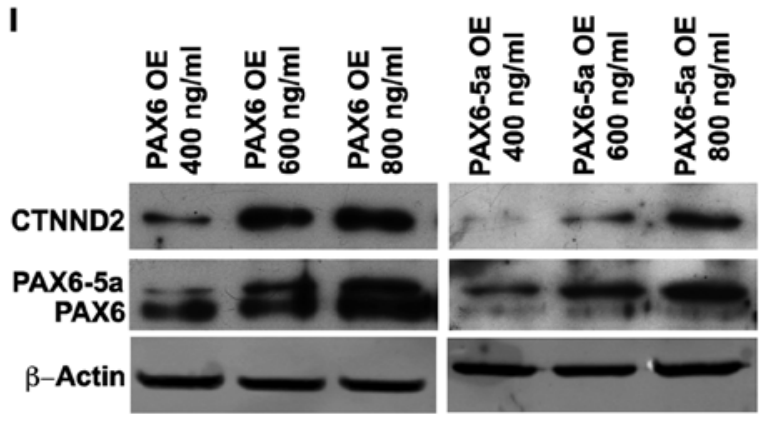

Figure 5. Regulation of PAX6 and PAX6-5a target genes in glioma cell lines. (A and B) qRT-PCR and (C) western blot analysis of the expression levels of PDGF-B, PAX6 and PAX6-5a in U87MG glioma cells, after OE of PAX6 or PAX6-5a. Evidently, PDGF-B is highly upregulated in the PAX6-5a OE cells (B and C), while a mild upregulation is also observed when PAX6 is overexpressed, possibly resulting from the observed simultaneous increase of PAX6-5a levels due to PAX6 OE. (C) Western blot analysis of SPHK1 after Pax6 or PAX6-5a OE. (D) qRT-PCR analysis of the levels of SPHK1 under this conditions, shows decreased SPHK1 expression after expression of both PAX6 isoforms, however most prominently under PAX6-5a overexpression (P<0.05). (E-I) qRT-PCR and western blot analysis of CTNDD2 expression after PAX6 or PAX6-5a OE, shows positive regulation of CTNND2 by PAX6-5a. This is evident only under moderate ( $<6$-fold increase) PAX6-5a OE conditions. When PAX6-5a is expressed at higher levels ( $>8$-fold), CTNDD2 is either diminished or not affected, compared to the control situation. PAX6 OE also results in a mild but significant upregulation of CTNDD2, however the effect could possibly result from the observed simultaneous increase in PAX6-5a levels ( $\left(\mathrm{P}<0.01 ;{ }^{* * *} \mathrm{P}<0.05\right.$; Error bars represent SEM from 3-4 independent experiments).

of its activity, respectively, suggesting that Pax6-5a exerts even a slightly greater regulatory potential as compared to PAX6
(Fig. 5D). Similar results were obtained at the protein level as shown on Fig. 5C, where OE of either PAX6 isoform, results 
in diminished SPHK1 protein levels. The greater reduction achieved after PAX6-5a OE, points towards a theoretically greater regulatory potential of this isoform to SPHK1.

The investigation of the regulatory relationship between the PAX6 isoforms and CTNDD2, proved more difficult because of the complex regulatory feedback loop between these genes (45). When PAX6 is 'moderately' overexpressed (less than 7-fold), then PAX6-5a and CTNND2 are also 'moderately' upregulated $(3.9 \pm 0.21$ and $3.85 \pm 0.65$-fold, respectively) (Fig. 5E). Higher OE of $P A X 6$ (9.8 \pm 0.59 -fold) results in higher expression (6.98 \pm 0.16 -fold) of $P A X 6-5 a$, however CTNND2 levels are only very mildly up (4.28 \pm 0.48 -fold) compare to the moderate PAX6 OE situation (Fig. 5F). One possibility could be that $P A X 6$ alone is not sufficient to promote higher upregulation of CTNND2 or alternatively, that $P A X 6-5 \mathrm{a}$ is the main regulator of $C T N N D 2$ and the upregulation seen under $P A X 6 \mathrm{OE}$ conditions is due to the simultaneous upregulation of PAX6-5a. For clarification, we overexpressed PAX6-5a at 'moderate' or 'high' levels in U87 cells. A 'moderate' OE of PAX6-5a (5.6 \pm 0.19 -fold) results in only 'mild' upregulation of PAX6 (2.45 \pm 0.19 -fold), while CTNND2 is upregulated by 4.2 \pm 0.23 -fold (Fig. 5G). Nevertheless, a higher (8.78 \pm 0.39 -fold) OE of PAX6-5a results in insignificant $(1.68 \pm 0.14$-fold $)$ upregulation of PAX6, but even more interestingly the expression of CTNDD2 is borderline downregulated (no change). This finding suggests that the levels of $P A X 6-5 a$ as well as the $P A X 6 / P A X 6-5 a$ ratio, is important for the passage from positive to negative regulation of CTNND2 (Fig. 5H). The results from WB analyses follow the same pattern described above under three different concentrations of PAX6 or PAX6-5a expression plasmid transfection (Fig. 5I).

Overexpression of either CTNND2 or SPHK1, in glioma cells, disrupts proliferation and promotes cell death. As shown in Fig. 3, OE of either PAX6 or PAX6-5a in glioma cell lines leads to decreased proliferation and increased apoptosis. This is in accordance with previous studies showing that conditional activation of the two isoforms during corticogenesis in vivo, results in defects in cell cycle progression (inhibition of proliferation) and in the acquisition of apoptotic or neuronal cell fate of the progenitors (11). In order to verify whether this is the result of the de-regulation of the downstream targets CTNND2 and of PAX6/PAX6-5a, we overexpressed them in U87 glioma cells and $48 \mathrm{~h}$ later we conducted immunostainings with Ki67.

As shown in Fig. 6A and B, in the control (GFP-vector) situation (where PAX6 and PAX6-5a are already expressed at insignificant levels due to their downregulation by enhanced expression of the miR-183-96-182 cluster) the $\mathrm{GFP}^{+}$cells expressing $\mathrm{Ki} 67$ reach almost $100 \%$. Under CTNND2-GFP $\mathrm{OE}$, less than half $(32.8 \pm 2.6 \%)$ of the $\mathrm{GFP}^{+}$cells express Ki67. Similarly, when SPHK1 is overexpressed, then the fraction of $\mathrm{SPHK} 1-\mathrm{GFP}^{+} / \mathrm{Ki}^{+} 7^{+}$cells become only $40 \pm 1.89 \%$ ). Caspase $3 / 7$ activity measurement also points towards a significant increase of cell death when CTNND2 is overexpressed; however, SPHK1 OE leads to only a mild increase of caspase 3/7 activity (Fig. 6C). Thus, although less than half of the SPHK $1 / \mathrm{GFP}^{+}$cells are proliferating, only a fraction of the non-proliferating cells dies. On the other hand, PDGF-B $\mathrm{OE}$, results in no significant change in the proliferative or apoptotic status of the glioma cells (Fig. 6A-C). The latter is somewhat expected, since U87 glioma cells already express high PDGF-B levels.

\section{Discussion}

Understanding the complex molecular mechanisms underlying cellular processes in cancer, is of great importance in order to decipher how tumors develop and progress. In this study, we unveiled the existence of cross-regulatory interactions between the two, active in vertebrate CNS, PAX6 isoforms, PAX6 and $P A X 6-5 a$, with the miR183-96-182 cluster that appears to play an essential role in glioma progression. Moreover, we found that the expression levels of miR-183-96-182 and PAX6/PAX6-5a could be valuable prognostic markers for disease outcome in human probands.

The results from our qRT-PCR analysis from 29 human glioma tumor biopsies and 3 glioma cell lines, revealed a significantly great reduction of both PAX6 isoform expression levels, as glioma progresses from low- to high-grade. On the contrary, members of the miR-183-96-182 cluster showed the exact opposite expression pattern, thus indicating a possible regulatory relationship between the miRNAs and TFs PAX6/PAX6-5a. Frequently miRNAs and TFs participate in feed-forward and feedback loop regulatory networks $(37,38)$ so that cellular processes are fine-tuned and temporally controlled. Indicated by luciferase reporter assays and bioinformatics analyses (data not shown), a feedback positive regulation of miR-183-96-182 by TFs PAX6/PAX6-5a might also exist, adding further complexity to the regulation of glioma tumor progression. However, this remains to be extensively investigated. Importantly, we found that different members of the miR-183-96-182 cluster regulate specifically the expression of either PAX6 or PAX6-5a. To our knowledge, such a preferential regulation of one or the other isoform of a TF by members of the same polycistronic, paralogous miRNA cluster, is a novel observation, concerning the basic mechanisms governing miRNA function.

Multiple studies have shown the involvement of the canonical PAX6 isoform in NSC proliferation, neurogenesis, generation of specific types of neurons, and changes in spatial pattern $(15,16,21,47)$. Measured by migration, invasion and survival assays, the OE of either PAX6 or PAX6-5a in high-grade glioma cells leads to decreased tumorigenicity, as opposed to the OE of miR-183-96-182, disclosing the important role of both PAX6 isoforms in restricting glioma progression. Our results are in accordance with previous findings reporting that PAX6-deficient cultured astrocytes display higher migration potential, which along with other capacities that they acquire, they resemble highly malignant glioma cells (48). Importantly, we were able to show that the expression of both PAX6 isoforms has a marked effect on glioma cell proliferation and death, however PAX6-5a exerts a more intense influence in reversing tumorigenicity. These findings, demonstrate for the first time that PAX6-5a may actually be the key isoform functioning in the early stages of glioma development to inhibit cell proliferation and promote glioma cell death.

Although high level of PAX6 has been reported to correlate with a better patient prognosis, the involved mechanism is still 
A
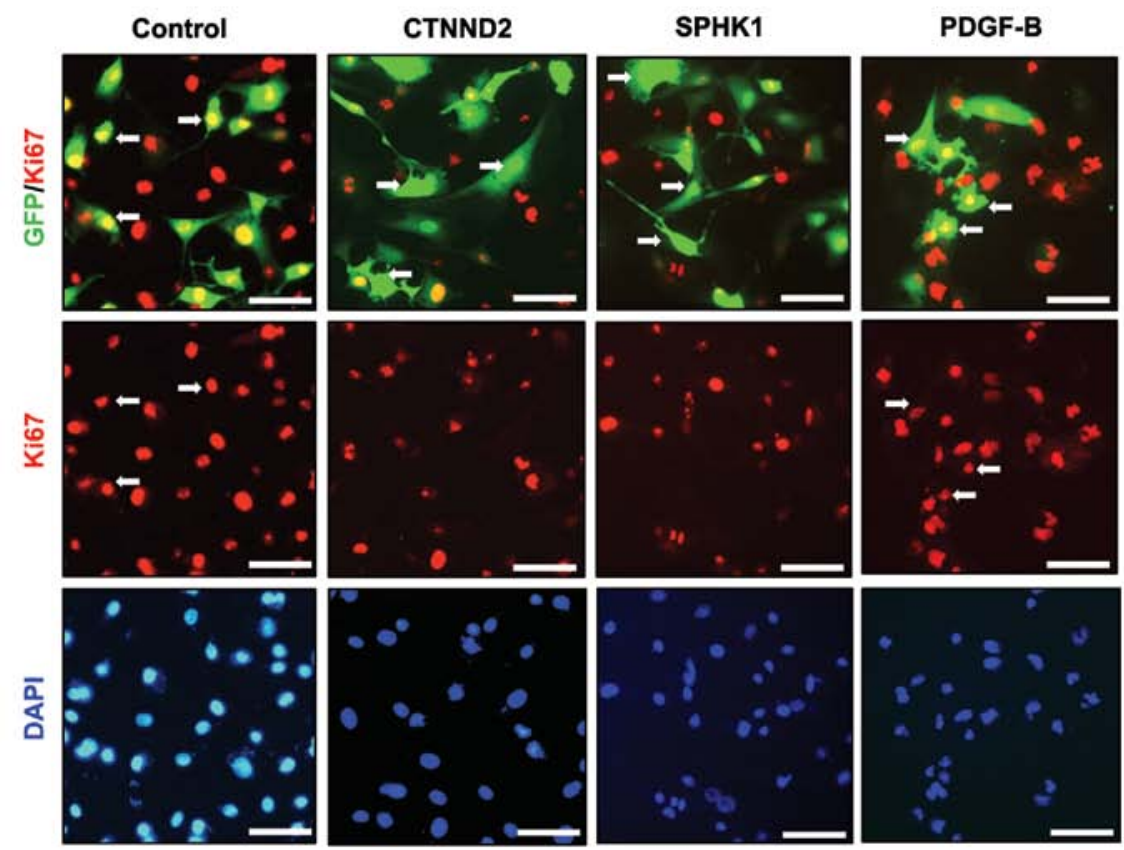

B

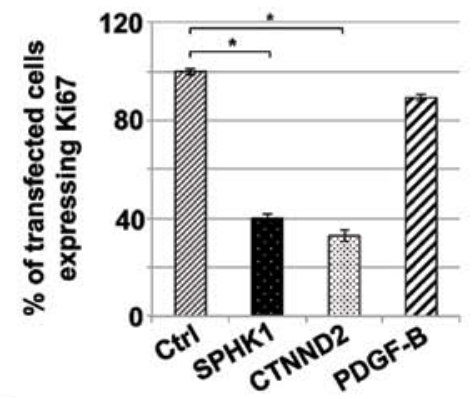

C

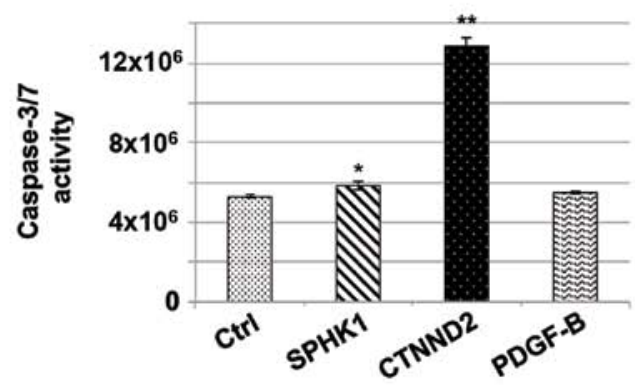

D

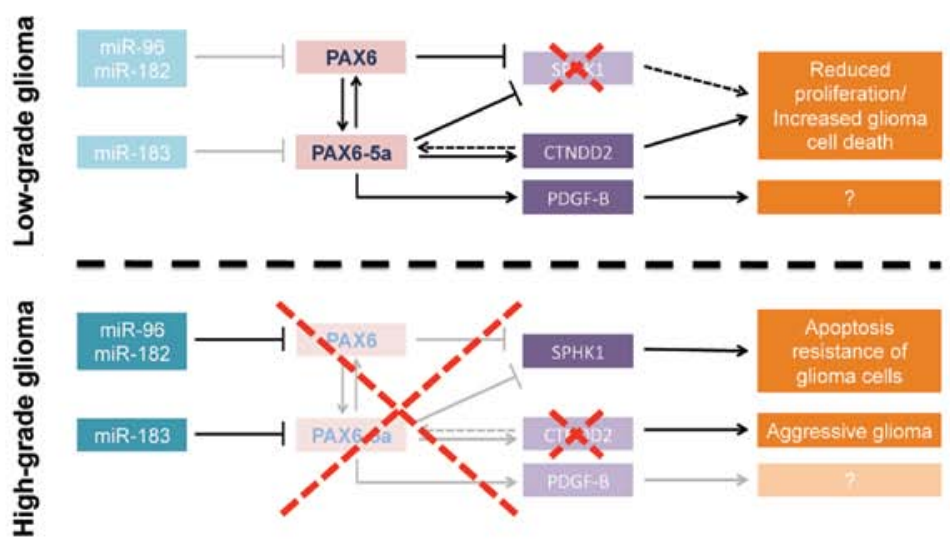

Figure 6. The PAX6 and PAX6-5a target genes CTNND2, SPHK1 and PDGF-B inhibit proliferation of glioma cells. (A) Representative immunofluorescence images of glioma cells following transfection of pCIG2-GFP control vector, CTNND2-GFP, SPHK1-GFP and PDGF-B-GFP, showing differences in the proliferation, as indicated by Ki67 immunostaining (red). (B) Counting of $\mathrm{GFP}^{+} / \mathrm{Ki} 67^{+}$glioma cells, under the same conditions, specifies a significantly ( $\mathrm{P}<0.01$ ) decreased cell proliferation after OE of CTNND2-GFP and SPHK1-GFP, while PDGF-B-GFP transfection displays no significant changes (Error bars show SEM from 3 independent transfections). (C) Measurement of caspase 3/7 activity of those transfected U87MG glioma cells, indicates increased rates of cell death after CTNND2-GFP ( $\left.{ }^{*}<0.01\right)$ and SPHK1-GFP $\left.{ }^{* *} \mathrm{P}<0.05\right)$ OE (Error bars show SEM from 3 independent transfections) (Scale bars: 100 $\mu$ m). (D) Simplified schematic illustration of our proposed theory of PAX6/PAX6-5a regulation in glioma. The miR-183-96-182 cluster downregulates TFs PAX6 and PAX6-5a, while glioma progresses from lower (I-II) to higher grades (III-IV). While all miRNAs of the cluster have the capacity to regulate both PAX6 isoforms, however, there is a strong tendency for higher specificity between miR-183 and PAX6-5a and miR-96-182 and PAX6. In low-grade glioma, the expression levels of miR-183-96-182 (faint blocks) are extremely low and thus not enough to control PAX6/PAX6-5a. As a result both isoforms are available in high quantities. PAX6/PAX6-5a block (red dashed X) the expression of its putative downstream target SPHK1, resulting in decreased glioma cell proliferation-increased cell death. Simultaneously, PAX6-5a induces CTNND2, which has anti-oncogenic function, to be expressed at higher levels. PDGF-B is also induced by PAX6-5a but the effect that this interaction has on glioma progression remains elusive. In high-grade glioma (GBM), the miR-183-96-182 are highly expressed. As a result both PAX6 and PAX6-5a isoforms are strongly downregulated and can no longer control the expression of their downstream effectors. SPHK1 is no longer blocked and its levels increase significantly leading to apoptosis resistance of the glioma cells. CTNND2 expression diminishes, resulting in aggressive glioma phenotype. PDGF-B is also reduced but as before, we have no significant data as to what effect this might have for the glioma progression. ( $\longrightarrow$, indicates inhibition or downregulation; black arrows indicate induction or upregulation; dashed black arrows indicate milder effect). 
unknown $(19,20)$. The performed qRT-PCR experiments on human tumor biopsies, resulted in the identification of at least 3 important candidates acting downstream of PAX6/PAX6-5a in glioma development, namely $S P H K 1, C T N N D 2$ and $P D G F-B$. Notably, while $S P H K 1$ was negatively regulated by both PAX6 isoforms, $P D G F-B$ was positively affected only by PAX6-5a. A complex cross-regulatory interaction of PAX6-5a and CTNND2, is already known (45), however we showed that a similar feedback regulation also occurs in gliomas. Intriguingly, our analysis indicates that the upregulation of CTNND2 by PAX6-5a and the simultaneous down-modulation of SPHK1 by both PAX6 isoforms, results in reduced glioma cell proliferation and elevated cell death, thus providing a molecular explanation of how these genes are controlled in relation to glioma progression.

To our knowledge, a specific, if any, association between PDGF-B and PAX6-5a has not been demonstrated. We found that PDGF-B is specifically upregulated by PAX6-5a. Nevertheless in glioma cells, high PDGF-B expression does not result in decreased proliferation/increased cell death. Previous studies suggested that overexpression of PDGF and corresponding receptors plays a role in development of CNS tumors $(4,5)$. In an in vivo model PDGF-B induced oligodendroglioma, PAX6 and the bHLH TF OLIG2, antagonize each other, where high PAX6 expression results in downregulation of OLIG2 and a less malignant phenotype (49). What is interesting though is that mice overexpressing PDGF-B alone do not form tumors, whereas they do so only when they are crossed with mice bearing mutations in other oncogenes (e.g. Tp53 null mice) (5).

The present results highlight a novel aspect for direct and specific regulation of the expression of the two PAX6 isoforms by the members of the miR-183-96-182 cluster. We show that high expression levels of both PAX6 and PAX6-5a result in reduced tumorigenicity of glioma cells, more prominently for isoform PAX6-5a, which is due to deregulated proliferation and increased cell death. Given the specific direct interaction of members of miR183-96-182 cluster with PAX6 and PAX6-5a, our findings argue towards the use of the clustered miRNAs, as well as the two PAX6 isoforms, as negative or positive markers, respectively, for glioma progression. Another line of presented evidence suggests that $S P H K 1, C T N N D 2$ and $P D G F-B$ could be PAX6/PAX6-5a downstream target genes. We herein show that elevated expression of $C T N N D 2$ or SPHK1 in glioma cells results in a similar to PAX6/PAX6-5a OE phenotype, leading to reduced cell growth in both cases and increased cell death in the case of CTNND2. Together, our findings suggest that the molecular interaction between PAX6 and PAX6-5a with specific members of the miR-18396-182 cluster plays an essential role in progression of glioma, in glioma cell lines, delivering their anti-tumorigenic effect, through the regulation of specific downstream pathways.

\section{Acknowledgements}

The authors wish to thank Silke Schlot and Martina Daniel for excellent technical assistance. The study was funded by Max Planck Gesellschaft and Cluster of Excellence and DFG Research Center for Nanoscale Microscopy and Molecular Physiology of the Brain (CNMPB).

\section{References}

1. Furnari FB, Fenton T, Bachoo RM, Mukasa A, Stommel JM, Stegh A, Hahn WC, Ligon KL, Louis DN, Brennan C, et al: Malignant astrocytic glioma: Genetics, biology, and paths to treatment. Genes Dev 21: 2683-2710, 2007.

2. Louis DN, Ohgaki H, Wiestler OD, Cavenee WK, Burger PC, Jouvet A, Scheithauer BW and Kleihues P: The 2007 WHO classification of tumours of the central nervous system. Acta Neuropathol 114: 97-109, 2007.

3. Adamson C, Kanu OO, Mehta AI, Di C, Li N, Mattox AK and Bigner DD: Glioblastoma multiforme: A review of where we have been and where we are going. Expert Opin Investig Drugs 18: 1061-1083, 2009.

4. Siebzehnrubl FA, Reynolds BA, Vescovi A, Steindler DA and Deleyrolle LP: The origins of glioma: E Pluribus Unum? Glia 59: 1135-1147, 2011.

5. Nazarenko I, Hede SM, He X, Hedrén A, Thompson J, Lindström MS and Nistér M: PDGF and PDGF receptors in glioma. Ups J Med Sci 117: 99-112, 2012.

6. Koul D: PTEN signaling pathways in glioblastoma. Cancer Biol Ther 7: 1321-1325, 2008.

7. Wang SI, Puc J, Li J, Bruce JN, Cairns P, Sidransky D and Parsons R: Somatic mutations of PTEN in glioblastoma multiforme. Cancer Res 57: 4183-4186, 1997.

8. Chang JY, Hu Y, Siegel E, Stanley L and Zhou YH: PAX6 increases glioma cell susceptibility to detachment and oxidative stress. J Neurooncol 84: 9-19, 2007.

9. Jacques TS, Swales A, Brzozowski MJ, Henriquez NV, Linehan JM, Mirzadeh Z, O' Malley C, Naumann H, AlvarezBuylla $\mathrm{A}$ and Brandner S: Combinations of genetic mutations in the adult neural stem cell compartment determine brain tumour phenotypes. EMBO J 29: 222-235, 2010.

10. Sanai N, Alvarez-Buylla A and Berger MS: Neural stem cells and the origin of gliomas. N Engl J Med 353: 811-822, 2005.

11. Berger J, Berger S, Tuoc TC, D'Amelio M, Cecconi F, Gorski JA, Jones KR, Gruss P and Stoykova A: Conditional activation of Pax6 in the developing cortex of transgenic mice causes progenitor apoptosis. Development 134: 1311-1322, 2007.

12. Götz M, Stoykova A and Gruss P: Pax6 controls radial glia differentiation in the cerebral cortex. Neuron 21: 1031-1044, 1998.

13. Heins N, Malatesta P, Cecconi F, Nakafuku M, Tucker KL, Hack MA, Chapouton P, Barde YA and Götz M: Glial cells generate neurons: The role of the transcription factor Pax6. Nat Neurosci 5: 308-315, 2002.

14. Osumi N, Shinohara H, Numayama-Tsuruta K and Maekawa M: Concise review: Pax6 transcription factor contributes to both embryonic and adult neurogenesis as a multifunctional regulator. Stem Cells 26: 1663-1672, 2008.

15. Quinn JC, Molinek M, Martynoga BS, Zaki PA, Faedo A, Bulfone A, Hevner RF, West JD and Price DJ: Pax6 controls cerebral cortical cell number by regulating exit from the cell cycle and specifies cortical cell identity by a cell autonomous mechanism. Dev Biol 302: 50-65, 2007.

16. Warren N, Caric D, Pratt T, Clausen JA, Asavaritikrai P, Mason JO, Hill RE and Price DJ: The transcription factor, Pax6, is required for cell proliferation and differentiation in the developing cerebral cortex. Cereb Cortex 9: 627-635, 1999.

17. Haubst N, Berger J, Radjendirane V, Graw J, Favor J, Saunders GF, Stoykova A and Götz M: Molecular dissection of Pax6 function: The specific roles of the paired domain and homeodomain in brain development. Development 131: 6131-6140, 2004.

18. Shimizu N, Watanabe H, Kubota J, Wu J, Saito R, Yokoi T, Era T, Iwatsubo $\mathrm{T}$, Watanabe $\mathrm{T}$, Nishina $\mathrm{S}$, et al: Pax6-5a promotes neuronal differentiation of murine embryonic stem cells. Biol Pharm Bull 32: 999-1003, 2009.

19. Zhou YH, Tan F, Hess KR and Yung WK: The expression of PAX6, PTEN, vascular endothelial growth factor, and epidermal growth factor receptor in gliomas: Relationship to tumor grade and survival. Clin Cancer Res 9: 3369-3375, 2003.

20. Zhou YH, Wu X, Tan F, Shi YX, Glass T, Liu TJ, Wathen K, Hess KR, Gumin J, Lang F, et al: PAX6 suppresses growth of human glioblastoma cells. J Neurooncol 71: 223-229, 2005.

21. Sansom SN, Griffiths DS, Faedo A, Kleinjan DJ, Ruan Y, Smith J, van Heyningen V, Rubenstein JL and Livesey FJ: The level of the transcription factor Pax6 is essential for controlling the balance between neural stem cell self-renewal and neurogenesis. PLoS Genet 5: e1000511, 2009. 
22. Esquela-Kerscher A and Slack FJ: Oncomirs - microRNAs with a role in cancer. Nat Rev Cancer 6: 259-269, 2006.

23. Garzon R, Calin GA and Croce CM: MicroRNAs in cancer. Annu Rev Med 60: 167-179, 2009.

24. Lavon I, Zrihan D, Granit A, Einstein O, Fainstein N, Cohen MA, Cohen MA, Zelikovitch B, Shoshan Y, Spektor S, et al: Gliomas display a microRNA expression profile reminiscent of neural precursor cells. Neuro Oncol 12: 422-433, 2010.

25. Li H, Kloosterman W and Fekete DM: MicroRNA-183 family members regulate sensorineural fates in the inner ear. J Neurosci 30: 3254-3263, 2010.

26. Xu S, Witmer PD, Lumayag S, Kovacs B and Valle D: MicroRNA (miRNA) transcriptome of mouse retina and identification of a sensory organ-specific miRNA cluster. J Biol Chem 282: 25053-25066, 2007.

27. Fendler A, Jung M, Stephan C, Erbersdobler A, Jung K and Yousef GM: The antiapoptotic function of miR-96 in prostate cancer by inhibition of FOXO1. PLoS One 8: e80807, 2013.

28. Liu Z, Liu J, Segura MF, Shao C, Lee P, Gong Y, Hernando E and Wei JJ: MiR-182 overexpression in tumourigenesis of high-grade serous ovarian carcinoma. J Pathol 228: 204-215, 2012.

29. Mi D, Carr CB, Georgala PA, Huang YT, Manuel MN, Jeanes E, Niisato E, Sansom SN, Livesey FJ, Theil T, et al: Pax6 exerts regional control of cortical progenitor proliferation via direct repression of Cdk6 and hypophosphorylation of $\mathrm{pRb}$. Neuron 78 : 269-284, 2013

30. Moskwa P, Buffa FM, Pan Y, Panchakshari R, Gottipati P, Muschel RJ, Beech J, Kulshrestha R, Abdelmohsen K, Weinstock DM, et al: miR-182-mediated downregulation of BRCA1 impacts DNA repair and sensitivity to PARP inhibitors. Mol Cell 41: 210-220, 2011.

31. Song L, Liu L, Wu Z, Li Y, Ying Z, Lin C, Wu J, Hu B, Cheng SY, Li M, et al: TGF- $\beta$ induces miR-182 to sustain NF- $\kappa$ B activation in glioma subsets. J Clin Invest 122: 3563-3578, 2012.

32. Weeraratne SD, Amani V, Teider N, Pierre-Francois J, Winter D, Kye MJ, Sengupta S, Archer T, Remke M, Bai AH, et al: Pleiotropic effects of miR-183 96 182 converge to regulate cell survival, proliferation and migration in medulloblastoma. Acta Neuropathol 123: 539-552, 2012.

33. Jiang L, Mao P, Song L, Wu J, Huang J, Lin C, Yuan J, Qu L, Cheng SY and Li J: miR-182 as a prognostic marker for glioma progression and patient survival. Am J Pathol 177: 29-38, 2010.

34. Tanaka H, Sasayama T, Tanaka K, Nakamizo S, Nishihara M, Mizukawa K, Kohta M, Koyama J, Miyake S, Taniguchi M, et al: MicroRNA-183 upregulates HIF-1 $\alpha$ by targeting isocitrate dehydrogenase 2 (IDH2) in glioma cells. J Neurooncol 111: 273-283, 2013.

35. Tang H, Bian Y, Tu C, Wang Z, Yu Z, Liu Q, Xu G, Wu M and Li G: The miR-183/96/182 cluster regulates oxidative apoptosis and sensitizes cells to chemotherapy in gliomas. Curr Cancer Drug Targets 13: 221-231, 2013.

36. Paul V, Tonchev AB, Henningfeld KA, Pavlakis E, Rust B, Pieler T and Stoykova A: Scratch2 modulates neurogenesis and cell migration through antagonism of bHLH proteins in the developing neocortex. Cereb Cortex 24: 754-772, 2014.
37. Tsang J, Zhu J and van Oudenaarden A: MicroRNA-mediated feedback and feedforward loops are recurrent network motifs in mammals. Mol Cell 26: 753-767, 2007.

38. Zhang HM, Kuang S, Xiong X, Gao T, Liu C and Guo AY: Transcription factor and microRNA co-regulatory loops: Important regulatory motifs in biological processes and diseases. Brief Bioinform 16: 45-58, 2015.

39. Aota S, Nakajima N, Sakamoto R, Watanabe S, Ibaraki N and Okazaki K: Pax6 autoregulation mediated by direct interaction of Pax6 protein with the head surface ectoderm-specific enhancer of the mouse Pax6 gene. Dev Biol 257: 1-13, 2003.

40. Pinson J, Mason JO, Simpson TI and Price DJ: Regulation of the Pax6 : Pax6(5a) mRNA ratio in the developing mammalian brain. BMC Dev Biol 5: 13-17, 2005.

41. Pinson J, Simpson TI, Mason JO and Price DJ: Positive autoregulation of the transcription factor Pax6 in response to increased levels of either of its major isoforms, Pax6 or Pax6(5a), in cultured cells. BMC Dev Biol 6: 25-34, 2006.

42. Guan H, Song L, Cai J, Huang Y, Wu J, Yuan J, Li J and Li M: Sphingosine kinase 1 regulates the Akt/FOXO3a/Bim pathway and contributes to apoptosis resistance in glioma cells. PLoS One 6: e19946, 2011.

43. Kiselev Y, Eriksen TE, Forsdahl S, Nguyen LH and Mikkola I: 3T3 cell lines stably expressing Pax6 or Pax6(5a) - a new tool used for identification of common and isoform specific target genes. PLoS One 7: e31915, 2012.

44. Zhang H, Li W, Sun S, Yu S, Zhang M and Zou F: Inhibition of sphingosine kinase 1 suppresses proliferation of glioma cells under hypoxia by attenuating activity of extracellular signalregulated kinase. Cell Prolif 45: 167-175, 2012.

45. Zhang J, Lu JP, Suter DM, Krause KH, Fini ME, Chen B and $\mathrm{Lu} \mathrm{Q}$ : Isoform- and dose-sensitive feedback interactions between paired box 6 gene and delta-catenin in cell differentiation and death. Exp Cell Res 316: 1070-1081, 2010.

46. Frattini V, Trifonov V, Chan JM, Castano A, Lia M, Abate F, Keir ST, Ji AX, Zoppoli P, Niola F, et al: The integrated landscape of driver genomic alterations in glioblastoma. Nat Genet 45: 1141$1149,2013$.

47. Estivill-Torrus G, Pearson H, van Heyningen V, Price DJ and Rashbass P: Pax6 is required to regulate the cell cycle and the rate of progression from symmetrical to asymmetrical division in mammalian cortical progenitors. Development 129: 455-466, 2002.

48. Sakurai K and Osumi N: The neurogenesis-controlling factor, Pax6, inhibits proliferation and promotes maturation in murine astrocytes. J Neurosci 28: 4604-4612, 2008.

49. Appolloni I, Calzolari F, Barilari M, Terrile M, Daga A and Malatesta P: Antagonistic modulation of gliomagenesis by Pax6 and Olig2 in PDGF-induced oligodendroglioma. Int $\mathbf{J}$ Cancer 131: E1078-E1087, 2012. 